\title{
COMPLETIONS OF PARTIALLY ORDERED SETS AND UNIVERSAL ALGEBRAS
}

\author{
By \\ M. N. BLEICHER and H. SCHNEDER (Madison, Wisconsin, USA) \\ (Presented by L. RéDEr)
}

\section{$\S 1$. Introduction}

The results in this paper were motivated by a search for the answers to the following questions: When can a given partially ordered algebra be embedded in a complete partially ordered algebra in such a fashion that the original algebra is dense and that the operations acquire or maintain continuity properties? What type of identities are preserved under such embeddings?

Properties of extensions and completions do not depend on the algebraic structure but only on the partial order. Thus in $\S \S 2-4$ we consider extensions and completions of partially ordered sets.

We are led to the notions of order preserving mappings, order true mappings (2. 14), and abnormal extensions $Z(2.6)$ of a partially ordered set $X$. The analogue of the statement that a subset $X$ of a topological space $Z$ is dense in $Z$ is the statement that $Z$ is an abnormal extension of $X$. If $\alpha$ is an $X$ preserving order true mapping of an abnormal extension $Z$ of $X$ onto $Y$, then $Y$ is an abnormal extension of $\alpha(Y)$, and $\alpha$ preserves suprema and infima (2.27). We construct a partially ordered set $\hat{Z}=\{\hat{z}: z \in Z\}$ of equivalence classes (2.19) each class consisting of all elements which can possibly be identified under $X$ preserving order true mappings. We show that $|z| \leqq 3$, for all $z \in Z$ (2. 20 and 2.22). If $Y$ is an $X$ preserving order true image of $Z$, then the natural mapping $\gamma$ of $Z$ onto $Z$, which is order true, factors trough $Y(2.35)$ and $\hat{Y}$ is isomorphic to $\hat{Z}(2.36)$. In fact in the linearly ordered case $\hat{Z}$ is essentially the unique normal extension of $X(2.42)$.

The structure of abnormal extensions is completely determined in the case of a linearly ordered set $X(\S 4):$ A universal abnormal extension $\mathscr{F} \mathscr{F}(X)$ is constructed such that every other such extension is faithfully embeddable into it (4.14). The question of which extensions are completions is also solved in that it is shown that they are essentially homomorphic images of $\mathscr{F} \mathscr{F}(X)(4.11)$. It is further shown that there is a minimal completion, the normal completion (CLIFFORD [1]) which is both a homomorphic image of all completions and isomorphically embeddable in all completions.

In the case of partially ordered sets it is not known to us if a maximal abnormal completion exists.

Extensions $Y$ of $X$ in which every element of $Y$ is a supremum of a subset of $X$ are called superior extensions. The structure of superior completions of partially ordered sets is also given $(\S 3)$ : We construct a completion $\mathscr{I}(X)$ which is a superior extension of $X$. Every other superior extension is faithfully embeddable in $\mathscr{I}(X)$, (3.19). All superior extensions which are complete are essentially homomorphic images of $\mathscr{F}(X)(3.20)$. 
The next problem ( $\S \S 5-6$ ) considered is that of extending the algebraic structure of partially ordered universal algebras to the completions. If $(\Omega, S)$ is an universal algebra (with possibly infinitary operations) on $S$, then each operation $\omega \in \Omega$ can be extended to an operation on $\mathscr{I}(S)$.

Among all possible ways of extending those operations we construct one of particular importance. This method always yields operations which preserve suprema (5.13). These particular operations have the property that any other method of extending the operations yields a greater value when the operations are applied to a set of operands (5.15). Further we show that many of the identities which have held in $(\Omega, S)$ will remain valid in the extension (5.30). Given an identity on $(\Omega, S)$, we do not know necessary and sufficient conditions for this identity to remain valid in the extension $(\Omega, \mathscr{I}(S)$ ). However if $S$ is linearly ordered, all identities remain valid (6.2):

In the linearly ordered case it is possible to extend the operation to the universal abnormal completion $\mathscr{F} \mathscr{F}(X)$ (in many ways). There are two identity preserving methods of extending the operations which are of particular importance. One extension is lower semi-continuous, the other is upper semi-continuous (6.9). Unfortunately it is impossible in general to obtain a continuous extension.

CLIFFORD's theorem on normal completions of commutative semigroups (6.15) is obtained in a more general setting $(6.14)$ : We show that a linearly ordered universal algebra has a unique extension to the normal completion if and only if it has a continuous extension to that completion, and that all identities are preserved for that extension. We also obtain certain results on the nature of the identities necessary to define an abstract group and other particular algebraic structures. We further obtain some results on the monotonicity of the many possible defining operations for linearly ordered groups.

We append $\S 7$, in which free universal algebras are constructed, for the reader's convenience.

We mention below an alternate approach from which the same theory could be developed: Given a partially ordered universal algebra $(\Omega, S)$ the operation can be extended to the set of subsets of $S, 2^{S}(5.6)$. In this new algebra one can say two sets are equivalent if they are cofinal. This equivalence is in fact a congruence, and the factor algebra so obtained is naturally isomorphic to the superior extension of $(\Omega, S)$ to $\mathscr{I}(S)$.

\section{Partially ordered sets}

We begin with several definitions concerning both partially ordered and linearly ordered sets.

(2. 1) Defintion. Let $Z$. be a linearly ordered set. We define the order topology (KELLY [5] p. 57) to be the topology in which the intervals $(a, \infty)=\{z: z>a\}$ and $(-\infty, a)=\{z: z<a\}$ form a subbasis for the open sets.

(2. 2) Definttion. Let $X$ be a subset of a linearly ordered set $Z$. We call $X$ dense in $Z$ if and only if every nonempty subset of $Z$ which is open in the order topology meets $X$. 
(2.3) Definition. Let $Y$ be a subset of a partially ordered set $Z$. Then $z$ is the supremum of $Y(z=\sup Y)$ if and only if

(i) For each $y \in Y$, we have $z \geqq y$, and

(ii) If, $z^{\prime} \geqq y$ for all $y \in Y$, then $z^{\prime} \geqq z$.

The infimum of $(z=\inf Y)$ is defined by reserving the order symbols above. satisfies

(2.4) Remark. A supremum of $Y$, if it exists, is unique. If $z=\sup Y$, then $z$

(ii') If $z>w$, then there exists $y \in Y$ such that $w y$. But (i) and (ii') do not imply (ii). For example, let $Z=\{a, b, c\}$, with $a<b$ and $a<c$. Let $Y=\{a\}$, then both $b$ and $c$ satisfy (i) and (ii'), but neither satisfies (ii). If $\mathcal{Z}$ is linearly ordered, then (i) and (ii') are equivalent to (i) and (ii).

We introduce further definitions, some of which will not be used till the next section. However, since our definitions are all related, and some are required now, we prefer to state them together. Some similar notions have been considered by Clifford [1] and Fuchs [3], p. 178.

(2. 5) Definitions. Let $Z$ be a partially ordered set, and let $X$ be a subset of $Z$. Let $z \in Z$. Then

(1) $z$ is called a superior element of $X$ in $Z$ if and only if $z=\sup Y$, for some non-empty subset $Y$ of $X$.

(2) $z$ is called an inferior element of $X$ in $Z$ if and only if $z=\inf Y$, for some non-empty subset $Y$ of $X$.

(3) $z$ is called a normal element of $X$ in $Z$ if and only if $z$ is both a superior and an inferior element of $X$ in $Z$.

(2.6) Definitions. Let $Z$ be a partially ordered set, and let $X$ be a subset of $Z$. Then

(1) $Z$ is called an abnormal extension of $X$ if and only if each $z \in Z$ is either an inferior or a superior element of $X$ in $Z$.

(2) $Z$ is called a superior extension of $X$ if and only if each $z \in Z$ is a superior element of $X$ in $Z$.

(3) $Z$ is called an inferior extension of $X$ if and only if each $z \in Z$ is an inferior element of $X$ in $Z$.

(4) $Z$ is called a normal extension of $X$ if and only if each $z \in Z$ is a normal element of $X$ in $Z$.

(2.7) Notation AND REMARKs. Let $Z$ be an extension of a partially ordered set $X$. Let $z \in Z$. Set $U=U(X, z)=\{x \in X: x \geqq z\}, L=L(X, z)=\{x \in X: x \leqq z\}$, and $U_{\#}^{\#}=U^{\#}(X, z)=U \backslash\{z\}, L^{\#}=L^{\#}(X, z)=L \backslash\{z\}$.

(1) If $z \notin X$, then $U^{\#}=U, L^{\#}=L$.

(2) If $Z$ is an abnormal extension of $X$, then either $z=\inf U$ or $z=\sup L$.

(2.8) Notation: Let $Z$ be a partially ordered set. We use the symbols $z_{1} \| z_{2}$ to denote that $z_{1}$ is incomparable to $z_{2}$, i.e., neither $z_{1} \geqq z_{2}$ nor $z_{2} \geqq z_{1}$.

(2.9) Lemma. Let $Z$ be a partially ordered set which is an abnormal extension of a linearly ordered set $X$. Then $Z$ is linearly ordered.

Proof. We suppose $z_{1}, z_{2} \in Z$ and $z_{1} \| z_{2}$. We first show that there exists $x_{1} \in X$ such that $x_{1} \| z_{2}$. The element $z_{1}$ is either an inferior or a superior element of $X$ in $Z$. We argue the case when $z_{1}=\sup Y, Y \leqq X$. If there exists a $y \in Y$ with $z_{2} \leqq y$ then 
$z_{2} \leqq y \leqq z_{1}$. If for all $y \in Y$, we have $z_{2} \geqq y$ then, since $z_{1}$ is the supremum of $Y$, $z_{2} \geqq z_{1}$. Hence if $z_{1} \| z_{2}$, there must exist $x_{1} \in Y \leqq X$ such that $x_{1} \| z_{2}$.

A repetition of this argument with $x_{1}$ replacing $z_{2}$, shows that there exists $x_{2} \in X$ for which $x_{1} \| x_{2}$. This is a contradiction since $X$ is linearly ordered.

(2. 10) Lemama. Let $X$ be a subset of a linearly ordered set $Z$. Then $X$ is dense in $Z$ if and only if $Z$ is an abnormal extension of $X$.

Proor. Let $X$ be dense in $Z$ and let $z \in Z \backslash X$. We assume that $z$ is neither a maximal nor a minimal element of $Z$, and we show that $z$ is either a superior or inferior element of $X$ in $Z$. Let $L^{\#}=L^{\#}(X, z)$ and $U^{\#}=U^{\#}(X, z)$. Since $Z$ is linearly ordered, $L^{\# \cup U} U^{\#}=X$. Since $z$ is neither maximal nor minimal, and $X$ is dense in $Z$, it follows that $L^{\#}=X \cap(-\infty, z) \neq \varnothing$, and $U^{\#}=X \cap(z, \infty) \neq \varnothing$. If $z=\sup L^{\#}$ or $z=\inf U^{\# \text {, }}$ there is nothing to prove. We shall assume the contrary and derive a contradiction. In this case, since $z \neq \sup L^{\#}$, there exists an upper bound $l$ of $L^{\#}$ with $l<z$, and similarly there exists a lower bound $u$ of $U^{\#}$ with $u>z$. On the one hand, since $L^{\#} \cup U^{\#}=X,(l, u) \cap X=\varnothing$; on the other hand, by the denseness of $X$ in $Z,(l, u) \cap$ $\cap X \neq \varnothing$, a contradiction.

If $z$ is the maximal or minimal element in $Z$ we replace $(l, u)$ in the above argument by $(l, \infty)$ or $(-\infty, u)$ respectively, unless $Z=\{z\}$, in which case everything is trivial.

Conversely, let $Z$ be an abnormal extension of $X$. The open intervals, including the semi-infinite ones, are a basis for the order topology. Let $\left(z_{1}, z_{2}\right)$ be a nonempty open interval where possibly $z_{1}=-\infty$ or $z_{2}=+\infty$. It is sufficient to show that $\left(z_{1}, z_{2}\right) \cap X \neq \varnothing$. Let $z \in\left(z_{1}, z_{2}\right)$. If $z \in X$, there is nothing further to prove. Otherwise, we know that $z$ is either an inferior or superior element, and here argue the case in which $z$ is a superior element. In this case $z=\sup L^{\#}(X, z)$ and hence there exists an element $x \in L^{\#}(X, z)$ such that $z_{1}<x \leqq z$, whence $x \in\left(z_{1}, z_{2}\right)$ which establishes the proposition.

(2.11) Proposition. Let $X$ be a linearly ordered set. Then $Z$ is an abnormal extension of $X$ if and only if $Z$ is linearly ordered and $X$ is dense in $Z$.

Proof. Immediate from (2.9) and (2.10).

(2. 12) Definitions. Let $Z$ be a partially ordered set and let $z \in Z$. Then $z^{+}$ $\left(z^{-}\right)$is used to denote inf $U^{\#}(Z, z)\left(\sup L^{\#}(Z, z)\right)$ when and only when inf $U^{\#}\left(\sup L^{\#)}\right.$ exists and is distinct from $z$. The element $z^{+}$is called the successor or $z$ while $z^{-}$ is called the predecessor of $z$.

(2. 13) REMARK. The above definitions ensure that the successor and predecessor, if they exist, are unique, and that there is no element strictly between $z$ and $z^{+}$ or strictly between $z$ and $z^{-}$.

(2. 14) Definitions. Let $Z$ and $Y$ be partially ordered sets.

(1) A mapping $\alpha$ of $Z$ into $Y$ is called order preserving if and only if $z_{1}, z_{2} \in Z$, $z_{1} \geqq z_{2}$ implies $\alpha\left(z_{1}\right) \geqq \alpha\left(z_{2}\right)$.

(2) A mapping $\alpha$ of $Z$ into $Y$ is called order true if and only if $\alpha$ is order preserving and $\alpha\left(z_{1}\right)>\alpha\left(z_{2}\right)$ implies $z_{1}>z_{2}$.

(3) A mapping is called an order isomorphism if it is $1-1$ and order true.

(4) Let $X \subseteq Z$. An order true (order preserving) mapping $\alpha$ is called $X$ preserving if $\alpha$ restricted to $X$ is an order isomorphism. 
(2. 15) REMARKs. (1) If $Z$ is partially ordered, then the following are equivalent:

(i) $\alpha$ is order true.

(ii) $\alpha$ is order preserving and if $z_{1} \| z_{2}$ then either

$$
\alpha\left(z_{1}\right) \| \alpha\left(z_{2}\right) \text { or else } \dot{\alpha}\left(z_{1}\right)=\alpha\left(z_{2}\right) \text {. }
$$

(2) In the case when $Z$ is linearly ordered, the concepts order true and order preserving are equivalent since (ii) above is vacuously satisfied.

(3) If $\alpha$ is an order true mapping of $Z$ into $Y$ and $\beta$ is an order true mapping of $Y$ into $W$, then the composite map $\beta \alpha$ of $Z$ into $W$ is also order true.

(4) If $\alpha$ is $1-1$, then a necessary and sufficient condition for $\alpha$ to be an order isomorphism is that $\alpha\left(z_{1}\right) \leqq \alpha\left(z_{2}\right)$ if and only if $z_{1} \leqq z_{2}$.

(2.16) Definition. Let $Z$ be an extension of $X$. Let $w, z \in Z$. We shall say $w$ and $z$ are neighbors (in $Z$ over $X$ ) if

(i) either $w \notin X$ or $z \notin X$, and

(ii) either $w=z^{+}$and $z=w^{-}$, or $z=w^{+}$and $w=z^{-}$.

(2. 17) LEMMA. Let $Z$ be an abnormal extension of $X$, and let $z \in Z$. If $z^{+}$and $z^{-}$ both exist, then $z \in X$.

Proof. Suppose $z^{+}$and $z^{-}$both exist. Then $z^{+}$is a lower bound for $U^{\#}(X, z)$ and $z^{-}$is an upper bound for $L^{\#}(X, z)$. By (2.7.2) either $z=\inf U(X, z)$ or $z=$ $=\sup L(X, z)$. Hence $z \in X$, by (2.7.1).

The corollary below follows immediately from the fact that successors and predecessors are unique.

(2. 18) COROllary. If $z \in Z \backslash X$, then $z$ has at most one neighbor.

(2.19) Definition. Let $Z$ be an abnormal extension of $X$. We now define a class $\hat{Z}=\hat{Z}(X)$ of subsets of $Z$.

(i) If $x \in X$ let $\hat{x}$ consist of $x$ and all neighbors of $x$.

(ii) If, for some $x \in X, z \in \hat{x}$, then we define $\hat{z}=\hat{x}$.

(iii) If for all $x \in X, z \notin \hat{x}$, then $\hat{z}$ consists of $z$ and all neighbors of $z$.

(iv) $\hat{Z}(X)=\{\hat{z}: z \in Z\}$. Where no confusion can arise, we shall write $\hat{Z}$ in place of $\hat{Z}(X)$.

Thus we have collapsed to one element all classes $\hat{z}$.

(2. 20) REMARKs. Let $Z$ be an abnormal extension of $X$.

(1) If $z_{1}, z_{2}$ are neighbors, then $\hat{z}_{1}=\hat{z}_{2}$. This follows from (2. 18).

(2) It follows easily from (2. 18) and the fact that the relation of being neighbors is symmetric, that $\hat{Z}$ partitions $Z$.

(3) Note that for any $z \in Z, \hat{z}$ is linearly ordered and $\mid \hat{z} \subset \leqq 3$. If $|\hat{z}|=3$, then $\hat{z}=\hat{x}=\left\{x^{-}, x, x^{+}\right\}$where $x \in X, x^{-}, x^{+} \in Z \backslash X$, and $x^{-+}=x=x^{+-}$. If $|\hat{z}|=2$, then $\hat{z}=\left\{w, w^{+}\right\}$where $w \in Z$, either $w \notin X$ or $w^{+} \notin X$ and $w^{+-}=w$.

(4) If $Z$ is a superior extension of $X$, then
(a) $|\hat{z}| \leqq 2$,
(b) If $\hat{z} \cap X=\varnothing$, then $\hat{z}=\{z\}$,
(c) If $\hat{z} \cap X \neq \varnothing$, then $\max \hat{z} \in X$. 
(2. 21) LEMMA. Let $Z$ be an abnormal extension of a partially ordered set $X$, and let $\alpha$ be an $X$-preserving order true mapping of $Z$ into a set $Y$. If $z=\inf U$, where $U \subseteq X$, and $\alpha(z) \in \alpha(U)$ then $z \in U$.

Proof. Suppose $\alpha(z)=\alpha(u)$, where $u \in U$, and let $v \in U \backslash\{u\}$. Since $v \geqq z$, we have $\alpha(v) \geqq \alpha(z)=\alpha(u)$, and as $\alpha$ preserves $X$ it follows that $\alpha(v)>\alpha(u)$. Hence as $\alpha$ is order true, $v>u$, and hence $u=\inf U=z$.

(2. 22) THEOREM. Let $Z$ be an abnormal extension of a partially ordered set $X$. Let $\alpha$ be an $X$-preserving order true mapping of $Z$ into a set $Y$. Then and

(1) $z_{1} \| z_{2}$ if and only if $\alpha\left(z_{1}\right) \| \alpha\left(z_{2}\right)$,

(2) $\alpha^{-1}(\alpha(z)) \subseteq \hat{z}$.

Proof. We show in (i) below that $\alpha^{-1}(\alpha(z))$ is linearly ordered and that $\left|\alpha^{-1}(\alpha(z))\right| \leqq 3$. In (ii) we prove (1), and in (iii) we complete the proof of (2).

(i) Let $K=\alpha^{-1}(\alpha(z)), z \in Z$. Suppose that $z_{1} \in K, z_{1} \notin X$, and that $z_{1}$ is an inferior element of $X$ in $Z$, say $z_{1}=\inf U, U \subseteq X$. We shall show that $z_{1}=\sup K$. For, suppose $\alpha\left(z_{1}\right) \in \alpha(U)$. Then by Lemma (2. 21), $z_{1} \in U \subseteq X$, contrary to assumption Hence, for all $u \in U, \alpha\left(z_{1}\right)<\alpha(u)$. Let $y \in K$. Then $\alpha(y)=\alpha\left(z_{1}\right)<\alpha(u)$, whence $y<u$, for all $u \in U$. It follows that $y \leqq \inf U=z_{1}$. Hence $z_{1}=\sup K$. We now deduce that $K \backslash X$ contains at most one inferior element, $z_{1}$, and if such a $z_{1}$ exists, $z_{1}=\sup K$. Similarly, $K \backslash X$ contains at most one superior element $z_{2}$, and if such a $z_{2}$ exists, $z_{2}=\inf K$. Clearly $z_{2} \leqq z_{1}$. But as $\alpha$ is $1-1$ on $X, K \cap X$ contains at most one element, say $x$. If $z_{1}$ and such an $x$ exist, then $x<z_{1}$, if $z_{2}$ and $x$ exist then $z_{2}<x$. Hence $K=\alpha^{-1}(\alpha(z))$ is linearly ordered and $|K| \leqq 3$.

(ii) Since $\alpha$ is order preserving, it is immediate from the definition that $\alpha\left(z_{1}\right) \| \alpha\left(z_{2}\right)$ implies $z_{1} \| z_{2}$. Suppose $\alpha\left(z_{1}\right) \geqq \alpha\left(z_{2}\right)$. If $\alpha\left(z_{1}\right)>\alpha\left(z_{2}\right)$, then since $\alpha$ is order.true $z_{1}>z_{2}$. If $\alpha\left(z_{1}\right)=\alpha\left(z_{2}\right)$ then, since $\alpha^{-1}\left(\alpha\left(z_{1}\right)\right)$ is linearly ordered, $z_{1}$ and $z_{2}$ are comparable. Hence (1) follows.

(iii) Suppose, $w_{1}, w_{2}, w_{3} \in Z$. If $\alpha\left(w_{1}\right)=\alpha\left(w_{2}\right) \neq \alpha\left(w_{3}\right)$, then $w_{3}>w_{1}$ if and only if $w_{3}>w_{2}$, and, similarly, $w_{3}<w_{i}$ if and only if $w_{3}<w_{2}$. For if $w_{3}>w_{1}$ then $\alpha\left(w_{3}\right)>\alpha\left(w_{1}\right)$, whence $\alpha\left(w_{3}\right)>\alpha\left(w_{2}\right)$ and so $w_{3}>w_{2}$. It follows that if $w_{1}, w_{2}$ are in $K=\alpha^{-1}(\alpha(z))$ and are neighbors in $K$ over $X \cap K$, then $w_{1}, w_{2}$ are neighbors in $Z$ over $X$ and hence (2) follows.

(2. 23) REMARK. If $\alpha$ is a mapping from a partially ordered set $Z$ into a partially ordered set $Y$, then the following are equivalent:

(i) $\alpha$ is order true and $z_{1} \|_{2}$ if and only if $\alpha\left(z_{1}\right) \| \alpha\left(z_{2}\right)$.

(ii) $\alpha$ is order true and if $z_{1} \| z_{2}$ then $\alpha\left(z_{1}\right) \| \alpha\left(z_{2}\right)$.

(iii) $\alpha$ is order preserving and if $z_{1} \| z_{2}$ then $\alpha\left(z_{1}\right) \| \alpha\left(z_{2}\right)$.

(iv) $\alpha$ is order true and $\alpha^{-1}(y)$ is linearly ordered for each $y \in Y$.

(2. 24) Notation. If $Z$ is any set, we shall denote the identity mapping of $Z$ onto $Z$ by $\varepsilon \mid Z$. If $\alpha$ is any mapping defined on $Z$, and $U \subseteq Z$, then $\alpha \mid U$ denotes the restriction of $\alpha$ to $U$.

(2. 25) DEFInItion. If $\alpha$ is a mapping of a set $Z$ onto a set $Y$ we call the mapping $\beta$ of $Y$ into $Z$ a right inverse of $\alpha$ if and only if $\alpha \beta(y)=y$ for all $y \in Y$. That is, $\alpha \beta=\varepsilon \mid Y$.

Of course, a right inverse of $\alpha$ exists if and only if $\alpha$ is onto $Y$ and then $\beta$ is $1-1$. 
(2. 26) Lemma. Let $Z$ and $Y$ be partially ordered sets and let $\alpha$ be an orcler true mapping of $Z$ onto $Y$. Then every right inverse $\beta$ of $\alpha$ is an order isomorphism of $Y$ into $Z$.

PRoor. Let $y_{1}, y_{2} \in Y$, and suppose $y_{1}>y_{2}$. Since $\alpha \beta\left(y_{1}\right)=y_{1}>y_{2}=\alpha \beta\left(y_{2}\right)$ and $\alpha$ is order true, we infer that $\beta\left(y_{1}\right)>\beta\left(y_{2}\right)$. Clearly therefore, $y_{1} \geqq y_{2}$ implies that $\beta\left(y_{1}\right) \geqq \beta\left(y_{2}\right)$ and so $\beta$ is order preserving.

Now suppose that $\beta\left(y_{1}\right)>\beta\left(y_{2}\right)$. Then $y_{1}=\alpha \beta\left(y_{1}\right) \geqq \alpha \beta\left(y_{2}\right)=y_{2}$, since $\alpha$ is order preserving, whence $y_{1} \geqq y_{2}$. But, obviously, $y_{1} \neq y_{2}$; and so $y_{1}>y_{2}$. It follows that $\beta$ is order true and, of course, $1-1$.

(2. 27) Propdsition. Leit $Z$ be an abnormal extension of the partially ordered set $X$. Let $\alpha$ be an $X$-preserving order true mapping of $Z$ onto a partially ordered set $Y$. If $W \subseteq Z$, and $\sup W(\inf W)$ exists then $\sup \alpha(W)(\inf \alpha(W))$ exists and

$$
\alpha(\sup W)=\sup \alpha(W) \quad(\alpha(\inf W)=\inf \alpha(W))
$$

PRoof. Let $w=\sup W$. Since $\alpha$ is order preserving, clearly $\alpha(w)$ is an upper bound for $\alpha(W)$. Let $y$ be any upper bound for $\alpha(W)$. We shall show that $y \geqq \alpha(w)$. Let $z_{0}=\max \alpha^{-1}(y)$, which exists by (2.22.2) and (2.20.3). Let $u \in W$. If $\alpha\left(z_{0}\right)=$ $=y=\alpha(u)$, then by definition of $z_{0}, z_{0} \geqq u$. If $\alpha\left(z_{0}\right)=y>\alpha(u)$, then $z_{0}>u$, since $\alpha$ is order true. Hence $z_{0}$ is an upper bound for $W$, and so $z_{0} \geqq w=\sup W$. Since $\alpha$ is order preserving, $y=\alpha\left(z_{0}\right) \geqq \alpha(w)$. It follows that $\alpha(w)$ is the supremum of $\alpha(W)$.

(2. 28) Corollary. Let $Z$ be an abnormal (superior, inferior, normal) extension of a partially ordered set $X$, and let $\alpha$ be an $X$-preserving order true mapping of $Z$ on to a set $Y$. Then $Y$ is an abnormal (superior, inferior, normal) extension of $\alpha(X)$.

Proof. Let $z \in Z$ be a superior (inferior) element of $X$ in $Z$. Since $\alpha$ is onto $Y$, by $(2.27) \alpha(z)$ is a superior (inferior) element of $\alpha(X)$ in $Y$. It follows from the definition that $Y$ is an abnormal (etc.) extension of $\alpha(X)$.

(2. 29) Corollary. Let $Z$ and $Y$ be linearly ordered sets, and let $X$ be a dense subset of $Z$. If $\alpha$ is an $X$-preserving order preserving mapping from $Z$ onto $Y$, then $\alpha(X)$ is dense in $Y$.

Proof. Since $X$ is a dense subset of the linearly ordered set $Z$, it follows that $Z$ is an abnormal extension of $X$, by (2. 10). Since $Z$ is linearly ordered, it follows by (2. 15.2) that $\alpha$ is order true. Hence this corollary follows from (2. 28).

(2.30) Proposition. Let $Z$ be an abnormal extension of a partially ordered set $X$. Let $\alpha$ and $\beta$ be two $X$-preserving order true mappings of $Z$ onto $Y$. If $\alpha|X=\beta| X$, then $\alpha=\beta$.

Proof. Since $Z$ is an abnormal extension of $X$, for each $w \in Z$, either $w=\sup W$ or $w=\inf W$, where $W \subseteq X$. Hence by $(2.27) \alpha(w)=\sup \alpha(W)=\sup \beta(W)=\beta(w)$ or $\alpha(w)=\inf \alpha(W)=\inf \beta(W)=\beta(w)$ respectively.

(2.31) Proposition. Let $Z$ be an abnormal extension of $X$. Let $w, z_{1}, z_{2} \in Z$. If $\hat{w} \neq \hat{z}_{1}=\hat{z}_{2}$, then $w>z_{1}$ if and only if $w>z_{2}$, and similarly, $w<z_{1}$ if and only if $w<z_{2}$. 
Proof. Let $w>z_{1}$. If $z_{2} \leqq z_{1}$, then by transitivity, $w>z_{2}$. If $z_{2}>z_{1}$, then either $z_{2}=z_{1}^{+}$, or $z_{2}=z_{1}^{++}$. But $w>z_{1}$ implies $w \geqq z_{1}^{+}$. However, $w \neq z_{1}^{+}$, since, in this case $z_{1}^{+}, \in \hat{z}_{1}$. Hence $w>z_{1}^{+}$. If $z_{2}=z_{1}^{+}$, this completes the proof. If $z_{2}=z_{1}^{++}$, then $w \geqq z_{1}^{++}$, since $w>z_{1}^{+}$. Again we see that $w \neq z_{2}$, since $w \notin \hat{z}_{2}$. Hence $w>z_{2}$. The rest follows by symmetries.

(2.32) Definimion. (1) Let $Z$ be an abnormal extension of $X$. We order $\hat{Z}=\hat{Z}(X)$ as follows: Let $a, b \in \hat{Z}, a \neq b$. Then $a>b$ if and only if there exist $y \in a$, $z \in b$ such that $y>z$.

(2) The mapping given by $\gamma(z)=\hat{z}$, is called the natural mapping of $Z$ onto $\hat{Z}$.

(2.33) REMARKS. (1) In view of (2.31), the relation of (2.32.1) is well-defined and anti-symmetric.

(2) The set $\dot{Z}$ is partially ordered by the above relation.

(2.34) THEOREM. Let $Z$ be an abnormal extension of $X$. Let $\gamma$ be the natural mapping of $Z$ onto $\hat{Z}$. Then $\gamma$ is an $X$-preserving order true mapping.

Proof. Follows immediately from the above results.

(2.35) THEOREM. Let $Z$ be an abnormal extension of a partially ordered set $X$. Let $\alpha$ be an $X$-preserving order true mapping of $Z$ onto $Y$. Then:

(1) There exists an order true mapping $\beta$ of $Y$ onto $Z$ such that the natural mapping $\gamma$ factors: $\gamma=\beta \alpha$.

(2) The mapping $\beta$ has a right inverse $\lambda$ of $\hat{Z}$ into $Y$ such that $\lambda \gamma|X=\alpha| X$.

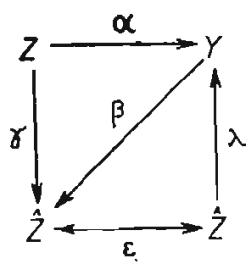

Comment. Intuitively, this theorem asserts that $\hat{Z}$ is the order true image of $Z$ which is minimal in two senses: (i) Every order true image of $Z$ in which $X$ is preserved can be mapped onto $\hat{Z}$ in an $X$-preserving order true fashion; and (ii) $\hat{Z}$ can be embedded order isomorphycally into every order true image of $Z$ in a fashion that preserves $X$.

Proof. (1) If $\alpha(z)=\alpha(1 v)$, then $\hat{z}=\hat{w}$ by (2.22. 2). Hence we may define a mapping $\beta$ of $Y$ onto $\hat{Z}$ by $\beta \alpha(z)=\hat{z}$, whence $\beta \alpha(z)=\gamma(z)$ and $\beta$ is $1-1$ on $\alpha(X)$.

We shall now show that $\beta$ is order true. Suppose $y_{1}=\alpha\left(z_{1}\right)>\alpha\left(z_{2}\right)=y_{2}$. Then $z_{1}>z_{2}$, since $\alpha$ is order true, and so $\beta\left(y_{1}\right)=\hat{z}_{1} \geqq \hat{z}_{2}=\beta\left(y_{2}\right)$ and so $\beta$ is order preserving. Next, suppose $\beta\left(y_{1}\right)>\beta\left(y_{2}\right)$. Therefore $\beta \alpha\left(z_{1}\right)>\beta \alpha\left(z_{2}\right)$ whence $\hat{z}_{1}>\hat{z}_{2}$. Thus $z_{1}>z_{2}$ and so $y_{1}=\alpha\left(z_{1}\right) \geqq \alpha\left(z_{2}\right)=y_{2}$. But $y_{1} \not \neq y_{2}$, hence $\beta$ is order true.

(2) By (2.28), $Y$ is an abnormal extension of $\alpha(X)$. We now define a mapping $\lambda$ of $Z$ into $Y$ which is a right inverse of $\beta$ as follows: For all $x \in X, \lambda(\hat{x})=\alpha(x)$. If $z \in Z$, and for all $x \in X, \hat{z} \neq \hat{x}$, then $\lambda(\hat{z})$ can be chosen to be any element $\alpha(w)$, 
with $w \in \hat{z}$. It is clear that $\lambda$ is a right inverse of $\beta$ and that $\lambda \gamma(x)=\lambda(\hat{x})=\alpha(x)$, for $x \in X$. By (2.26), $\lambda$ is order true.

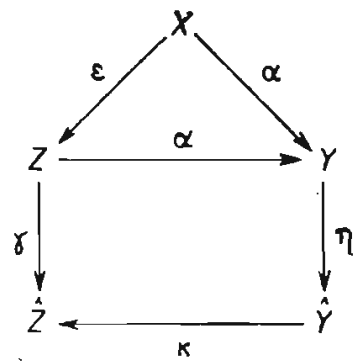

(2. 36) Proposition. Let $Z$ be an abnormal extension of a partially ordered set $X$. Let $\alpha$ be an order true mapping of $Z$ onto $Y$. Let $\gamma$ be the natural mapping of $Z$ onto $\hat{Z}(X)=\hat{Z}$ and let $\eta$ be the natural mapping of $Y$ onto $\hat{Y}(\alpha(X))=\hat{Y}$. Then there exists an order isomorphism $\varkappa$ of $\hat{Y}$ onto $\hat{Z}$ such that $\varkappa \eta \alpha=\gamma$.

Proor. Since $\eta \alpha$ is an $X$-preserving order true mapping of $Z$ onto $\hat{Y}$, by (2.35) there exists an order true mapping $x$ of $\hat{Y}$ onto $\hat{Z}$ such that $x \eta \alpha=\gamma$. We must show that $x$ is $1-1$. So let $\hat{y}_{1}, \hat{y}_{2} \in \hat{Y}$ and suppose $x\left(\hat{y}_{1}\right)=x\left(\hat{y}_{2}\right)$. Then $x \eta\left(y_{1}\right)=x \eta\left(y_{2}\right)$ and, as $\varkappa \eta$ is an $\alpha(X)$ preserving order true mapping from $Y, y_{1} \in(x \eta)^{-1}(x \eta)\left(y_{2}\right) \leqq \hat{y}_{2}$, by (2. 22. 2). Hence $\hat{y}_{1}=\hat{y}_{2}$, and the result is proved.

(2.37) COROLlary. Let $Z$ be an abnormal extension of a partially ordered set $X$. Let $\alpha$ be an $X$-preserving order true mapping of $Z$ onto $Y$. Then there is an order isomorphism $\beta$ of $Y$ onto $Z$ such that $\beta \alpha=\gamma$ if and only if for all $y \in Y, \hat{y}=\{y\}$.

Proof. By (2.35) there exists an order true mapping $\beta$ from $Y$ onto $Z$ such that $\beta \alpha=\gamma$, and since $\alpha$ is onto $Z$, this mapping $\beta$ is unique. Hence $\beta=\varkappa \eta$, where $x, \eta$ are defined in (2.36); and so $\beta$ is $1-1$ if and only if $\eta$ is $1-1$. But, by definition of $\hat{Y}, \eta$ is $1-1$ if and only if $\hat{y}=\{y\}$, for all $y \in Y$.

(2.38) COROLlary. Let $Z$ be an abnormal extension of a partially ordered set $X$. Then for all $a \in \hat{Z}, \hat{a}=\{a\}$, i.e. $\hat{\mathcal{Z}}$ is isomorphic to $\hat{Z}$ under the natural mapping.

Proof. Put $Y=Z, \alpha=\gamma$, and $\beta=\varepsilon$ in (2. 37).

(2.39) Proposition. Let $Z$ be a normal extension of a partially ordered set $X$. Then, for all $z \in Z, \hat{z}=\{z\}$.

Proor. Let $z_{1}, z_{2} \in Z$. We shall show that $z_{1}$ and $z_{2}$ are not neighbors. If both $z_{1} \in X$ and $z_{2} \in X$, then the conclusion follows immediately from definition (2.16). So suppose $z_{1} \notin X$. If $z_{1} \| z_{2}$, then again the conclusion follows. Suppose $z_{2}<z_{1}$. Since $Z$ is a normal extension of $X, z_{1}=\sup L^{\#}\left(X, z_{1}\right)$ whence by $(2.12) z_{1}^{-} \not \neq z_{2}$, and so $z_{1}$ is not a neighbor of $z_{2}$. The case $z_{1}<z_{2}$ is similar.

(2.40) Definition. I.et $S$ and $T$ be linearly ordered sets. Then $S$ and $T$ are of the same type (at infinity) if $S$ has a maximal element if and only if $T$ does, and $S$ has a minimal element if and only if $T$ does. 
(2. 41) Proposition. Let $Z$ be an abnormal extension of a linearly ordered set $X$. Then $Z$ is a normal extension of $X$ if and only if the natural map of $Z$ onto $\hat{Z}$ is an order isomorphism and $Z$ is of the same type as $X$.

Proof. Half the proposition follows from (2.39), and the fact that a normal extension must be the same type as the set it extends. Let the natural mapping $\gamma$ be $1-1$. Let $z \in Z$. If $z \in X$, then $z$ is a normal element. Suppose $z \in Z \backslash X$. Then $L^{\#}(X, z)=L(X, z)=L$, is non-empty, since $z$ is not minimal. If $z \neq \sup L$ then, since $X$ is linearly ordesed, sup $L$ exists and equals $z^{-}$, a neighbor of $z$. But this is impossible, since $\gamma$ is $1-1$. Hence $z=\sup L$, a superior element. Similarly $z$ is an inferior element, whence $z$ is normal.

(2. 42) Corollary. Let $X$ be a linearly ordered set and let $Z$ be an abnormal extension of $X$ of the same type as $X$. Then $\hat{Z}$ is a normal extension of $X$.

Proof. Follows from (2.38) and (2.41).

(2. 43) Lemma. Let $Z$ be an abnormal extension of $X$, and suppose $\alpha$ is an order true mapping of $Z$ into $Z$ which is the identity on $X$. Then

(1) $U(X, z) \subseteq U(X, \alpha(z))$. and $L(X, z) \subseteq L(X, \alpha(z))$.

(2) $\alpha(z)$ is comparable to $z$.

(3) $\alpha^{2}=\alpha$.

Proof. (1) Let $z \in Z$, and let $x \geqq z, x \in X$. Then $x=\alpha(x) \geqq \alpha(z)$, whence $U=$ $=U(X, z) \subseteq U(X, \alpha(z))$. Similarly, $L=L(X, z) \subseteq L(X, \alpha(z))$.

(2) Thus $\alpha(z)$ is botll a lower bound for $\bar{U}$, and an upper bound for $L$. Since either $z=\inf U$ or $z=\sup L$, it follows that $\alpha(z)$ and $z$ are comparable.

(3) If $\alpha(z) \in X$, or $\alpha(z)=z$, then obviously $\alpha^{2}(z)=\alpha(z)$. So suppose $\alpha(z) \notin X$, and $\alpha(z) \neq z$. Let $x \in X$ and $x \geqq \alpha(z)$. Then $x=\alpha(x)>\alpha(z)$, and since $\alpha$ is order true, $x>z$. Hence $U(X, z) \supseteqq U(X, \alpha(z))$. By $(1)$, we conclude that $U=U(X, z)=U(X, \alpha(z))$. Similarly $L=L(X, \alpha(\bar{z}))$. By (2) we may suppose without loss of generality, that $\alpha(z)=z$. Then $z=\sup L$ and $\alpha(z)=\inf U$. Further since $\alpha(z)>z$, we have $\alpha^{2}(z) \geqq \alpha(z)$. But $U\left(X, \alpha^{2}(z)\right) \supseteqq U(X, \alpha(z))$, and hence $\alpha^{2}(z)$ is lower bound for $U$, whence $\alpha^{2}(z) \leqq \alpha(z)$. Thus $\alpha^{2}(z)=\alpha(z)$.

(2. 44) Proposition. Let $Z$ be an abnormal extension of $X$. Let $\alpha$ be an order true mupping of $Z$ into $Z$ which is the identity on $X$. Then for all $z \in Z$, either $\alpha(z)=z$ or $\alpha(z)$ and $z$ are neighbors in $Z$ over $X$.

Proof. By (2.22.2) and (2.39) we obtain

$$
z \supseteqq \alpha^{-1}(\alpha(z))=\alpha^{-1}\left(\alpha^{2}(z)\right),
$$

whence $z, \alpha(z) \in \hat{z}$. If $z \cap X=\varnothing$, the result follows immediately from (2.20.3). If $\hat{z}=\left\{x^{-}, x, x^{+}\right\}, x \in X$ then either $z=\alpha(z)$ or $\alpha(z)$ must equal $x$, by $(2,43.1)$, whence $z$ and $\alpha(z)$ are neighbors.

(2. 45) COROLLARY. Let $\alpha$ be an order true mapping of $Z$ into itself which is the identity on $X$. Then for all $z \in Z, \alpha(z) \in \hat{z}$.

Proof. Immediate, by (2. 44). 
(2. 46) Lemma. Let $Z$ be an abnormal extension of a partially orderéd set $X$. Let $\alpha$ be an $X$-preserving order true mapping of $Z$ into $Y$. Let $\beta$ be an $\alpha(Z)$-preserving order true mapping of $Y$ into $Z$ such that $\beta \alpha|X=\varepsilon| X$. Then $\alpha \beta \alpha=\alpha$.

Proof. Let $\gamma=\beta \alpha$. Then $\gamma$ is an order true mapping of $Z$ into $Z$ which is the identity on $X$. By $(2.43 .3) \gamma^{2}=\gamma$, namely, $\beta \alpha \beta \alpha=\beta \alpha$. Since $\beta$ is $1-1$ on $\alpha(Z)$, we obtain $\alpha \beta \alpha=\alpha$.

(2. 47) Proposition. Let $Z$ be an abnormal extension of a partially ordered set $X$. Let $\alpha$ be an $X$-preserving order true mapping of $Z$ onto $Y$. Let $\beta$ be $a 1-1$ mapping of $Y$ into $Z$ such that $\beta \alpha|X=\varepsilon| X$. Then $\beta$ is order true if and only if $\beta$ is a right inverse of $\alpha$. Furthermore, such a right inverse $\beta$ exists.

Proof. By (2.26) if $\beta$ is a right inverse of $\alpha$, then $\beta$ is order true. Now suppose that $\beta$ is order true. By (2.46), $\alpha \beta \alpha=\alpha$. Let $y \in Y$. Since $\alpha$ is onto $Y$, we have that $y=\alpha(z)$, for some $z \in Z$. Hence $\alpha \beta(y)=\alpha \beta \alpha(z)=\alpha(z)=y$, and the equivalence of the two conditions follows. We can construct such a $\beta$ as follows: Let

$$
\beta(y)= \begin{cases}x, & \text { if } y=\alpha(x) \\ z, & \text { where } z \in \alpha^{-1}(y), \text { if } y \notin \alpha(X) .\end{cases}
$$

(2. 48) REMARKs. (1) A function $\beta$ may be effectively defined without the implicit use of the axiom of choice by setting $\beta(y)=\max \alpha^{-1}(y)$, for $y \notin \alpha(X)$, since $\alpha^{-1}(y)$ has at most two linearly ordered elements by (2.22.2) and (2.20.3).

(2) The number of distinct $\beta$ satisfying the conditions of (2. 43) is $\prod\left\{\left|\alpha^{-1}(y)\right|: y \in Y \backslash \alpha(X)\right\}$, which is always less than or equal to $2^{|Y| \alpha(X) \mid}$, since $\left|\alpha^{-1}(y)\right| \leqq 2$, if $y \in \alpha(X)$.

(2. 49) Proposition. Let $Z$ be an abnormal extension of $X$. Then $|Z| \leqq 2 \cdot\left(2^{|X|}-1\right)$.

PRoof. Since $Z$ is abnormal, each $z \in Z$ is either the supremum or the infimum of a non-empty subset of $X$. Since every subset of $X$ has at most one supremum and one infimum, we see that $|Z| \leqq 2 \cdot\left(\left|2^{X}\right|-1\right)=2 \cdot\left(2^{|X|}-1\right)$.

(2. 50) REMARK. The above is best possible in the sense that given any infinite cardinal number there is a partially ordered set of that cardinality for which equality holds.

\section{Superior completions}

(3.1) Definitions. Let $S$ be a partially ordered set. A subset $I$ of $S$ is called an initial segment of $S$ if $I$ is non-empty and if $s \in I$ and $t \leqq s$ imply that $t \in I$. A subset $F$ of $S$ is called a final segment of $S$ if $F$ is non-empty and if $s \in F$ and $t \geqq s$ imply that $t \in F$.

The set of all initial segments of $S$ will be denoted by $\mathscr{I}(S)$, the set of all final segments of $S$ by $\mathscr{F}(S)$.

(3. 2) Definitions. Let $S$ be a partially ordered set. If $s \in S$, then

$$
\begin{aligned}
\iota(s) & =\{t \in S: t \leqq s\}, & \iota^{-}(s) & =\{t \in S: t<s\}, \\
\varphi(s) & =\{t \in S: t \geqq s\}, & \varphi^{+}(s) & =\{t \in S: t>s\} .
\end{aligned}
$$


(3. 3) LEMMA. The set $\mathscr{I}(S)$ is partially ordered by inclusion. The mapping $\iota$ is an order true embedding of $S$ into $\mathscr{I}(S)$. If $S$ is linearly ordered, then $\mathscr{I}(S)$ is linearly ordered.

Proof. The proof is a straightforward verification.

(3. 4) Remarks. (1) Note that for all $s \in S, \iota(s) \in \mathscr{I}(S)$, and unless $s$ is a minimal element of $S, \iota^{-}(s) \in \mathscr{I}(S)$.

(2) We note that $\iota^{-}$is an order preserving mapping of $S$ into $\mathscr{I}^{\prime}(S) \cup\{\varnothing\}$, where $\varnothing$ is taken as the minimal element. However, $\iota^{-}$need not be $1-1$ or order true unless $S$ is linearly ordered.

(3) If $s$ is not a minimal element in $S$, then $\iota(s)^{-}$exists (cf. Definitions 2.12) and $\iota^{-}(s)=\iota(s)^{-}$. Further if $s^{-}$exists then also $\iota^{-}(s)=\iota\left(s^{-}\right)=\iota(s)^{-}$. If $s^{+}$exists then $\iota(s)=\iota^{-}\left(s^{+}\right)$, and if $\iota^{-}(s)^{+}$exists then $\iota(s)=\iota^{-}(s)^{+}$. Starting with these, one can prove inductively, under the proper existential hypotheses, that two expressions of the above type are equal whenever the differences between the number of plus signs and the number of minus signs in each expression are equal.

(4) If $\iota^{-}(s)=\iota(t)$, then $t=s^{-}$.

(3.5) Definition. Let $S$ be a partially ordered set. Then $S$ is (conditionally) complete if and only if every subset of $S$ which is bounded above has a supremum. (see Kelley [5], p. 14).

(3. 6) REMARK. A standard argument shows that $S$ is complete if and only if every subset of $S$ which is bounded below has an infimum.

(3. 7) Definition. Let $S$ be a partially ordered set. A complete set $T$ is called a completion of $S$ under $\alpha$ if and only if $\alpha$ is a 1-1 and order true mapping of $S$ into $T$. We call $T$ an abnormal completion of $S$ under $\alpha$ if and only if $T$ is a completion of $S$ under $\alpha$ which is an abnormal extension of $\alpha(S)$. Superior, inferior, and normal completions of $S$ under $\alpha$ are defined similarly.

If $\alpha$ is the identity mapping, we simply say $T$ is a completion of $S$.

(3. 8) Definition. Let $T$ be a partially ordered set. By $T_{+}$we mean $T$ if $\sup T=$ $=+\infty$, say, exists, and $T \cup\{+\infty\}$ if sup $T$ does not exist, where $+\infty$ is adjoined as the supremum of $T$. By $T_{-}$we mean $T$ if sup $T=-\infty$, say, exists, and $T \cup\{-\infty\}$ otherwise, where $-\infty$ is adjoined as the infimum of $T$. We define $T_{ \pm}=\left(T_{+}\right)_{-}=\left(T_{-}\right)_{+}$.

(3.9) Remark. If $T$ is complete, then every subset of $T_{+}$has a supremum in $T_{+}$, and every subset of $T_{-}$has an infmum in $T_{-}$. If $T$ is also linearly ordered, then $T_{ \pm}$is compact, and is usually called the two point compactification of $T$.

(3. 10) Lemma. For every partially ordered set $S$, the set $\mathscr{I}(S)$ is complete. If $\varnothing$ is adjoined as the minimal element of $\mathscr{I}(S)$, then $\mathscr{I}(S) \cup\{\varnothing\}$ is a lattice under union and intersection, complete in the latice theoretic sense and, for every $\mathscr{I} \subseteq \mathscr{J}(S) \cup\{\varnothing\}$

in $\mathscr{I}(S) \cup\{\varnothing\}$.

$$
\sup \mathscr{J}=\bigcup \mathscr{J}, \quad \text { inf } \mathscr{J}=\bigcap \mathscr{J}
$$

Proof. We note that the union of a set of initial subsets is initial, and the intersection of such a set is initial or empty. The lemma follows easily.

(3. 11) COROLlaRx. If $S$ is a linearly ordered set, then every closed subset of $\mathscr{I}(S)$ which is bounded below is compact in the order topology. 
Proof. We recall that a linearly ordered set is complete if and only if every closed bounded set is compact (KeLLy [5], p. 162). By (3. 3), $\mathscr{F}(S)$ is linearly ordered and by $(3.10), \mathscr{I}(S)$ is complete. Since, $\mathscr{I}(S)$ has $S$ as maximal element, every set which is bounded below is bounded, and the result follows. of $\iota(S)$.

(3. 12) LEMMA. If $S$ is a partially ordered set, then $\mathscr{I}(S)$ is a superior extension

Proof. Let $I \in \mathscr{I}(S)$. We note that for $s \in I$, we have $s \in \iota(s) \subseteq I$. By (3: 10) we obtain the equality

$$
I=\cup\{\iota(s): s \in I\}=\sup \{\iota(s): s \in I\}
$$

and the lemma follows.

The lemmas (3.3), (3.10) and (3.12) yield the following theorem.

(3.13) THEOREM. If $S$ is a partially ordered set, then $\mathscr{F}(S)$ is a superior completion of $S$ under $\iota$.

(3. 14) Corollary. If $S$ is linearly ordered, then $\iota(S)$ is dense in $\mathscr{F}(S)$ in the order topology.

Proof. Since a superior extension is abnormal, the corollary follows from (3. 13) and (2.10).

A dual theory can be developed for the set $\mathscr{F}(S)$ of final segments of $S$. The partial order is defined by $F_{1} \leqq F_{2}$ if and only if $F_{2} \subseteq F_{1}$. We state below the dual of (3.13). The proposition dual to $(m \cdot n)$ will be denoted by $(m \cdot n)^{*}$.

(3. 13)* Theorem. If $S$ is a partially ordered set then $\mathscr{F}(S)$ is an inferior completion of $S$ under $\varphi$.

Let us further note that in (3.10)* the infimum is the union, more precisely, if $\mathscr{K} \subseteq \mathscr{Z}(S)$ then inf $\mathscr{K}=\cup \mathscr{K}$. If $\mathscr{K}$ is bounded above, then sup $\mathscr{K}=\cap \mathscr{K}$.

(3. 15) EXAMPLEs. (1) Let $\mathbf{Z}$ be the set of integers. Then $\mathscr{I}(\mathbf{Z})=\iota(\mathbf{Z}) \cup\{\mathbf{Z}\}$, so that $\mathscr{I}(\mathbf{Z})$ is order isomorphic to $\mathbf{Z} \cup\{\cong \infty\}$.

(2) Let $\mathbf{R}$ be the set of real numbers. Then $\mathscr{I}(\mathbf{R})={ }^{\prime}(\mathbf{R}) \cup \iota-(\mathbf{R}) \cup\{\mathbf{R}\}$, where $\iota^{-}(r)$ is the immediate predecessor of $\iota(r)$. Thus $\mathscr{I}(\mathbf{R})$ is essentially the reals with positive infinite element and with each number split in two.

Example (3.15.2) is put into its proper perspective by the following proposition.

(3.16) Proposition. Let $S$ be a complete linearly ordered set, and let $S \uparrow$ be the set of non-minimal elements of $S$. Then $\mathscr{I}(S)=(S) \cup_{\iota}-(S \uparrow) \cup\{S\}$.

Proof. Clearly $\mathscr{I}(S) \supseteq \iota(S) \cup \iota^{-}\left(S_{\uparrow}\right) \cup\{S\}$. Let $I \in \mathscr{I}(S)$. Either $I=S$, or, since $I$ is initial, $I$ is bounded above. Since $S$ is complete, in this case $I$ has a supremum, say, $s$. Either $I=\iota(s)$ or $I=\iota^{-}(s)$, since $I$ is initial. If $I=\iota^{-}(s)$, then clearly $s \in S \uparrow$.

The following theorem is stated in terms of $\mathscr{F}(S)$ and $\varphi$, because this is the more natural setting for this result.

(3. 17)* THEOREM. Let $S$ be a partially ordered set. Then $\varphi(S)=\mathscr{F}(S)$ if and only if $S$ is well-ordered.

Proof. Suppose $\varphi(S)=\mathscr{F}(S)$, and let $F \in \mathscr{F}(S)$. By hypothesis, there exists an $s \in S$ such that $F=\varphi(s)$, and this $s$ is clearly the unique minimal element of $F$. 
Let $G \subseteq S$. Let $F=\bigcup\{\varphi(g): g \in G\}$. Then $F \in \mathscr{F}$ and so has a unique minimal element $t$, which is also the unique minimal element of $G$. Thus $S$ is well-ordered. On the other hand, if $S$ is well-ordered and $F \in \mathscr{F}$, then $F$ has an unique minimal element $s$, for which $F=\varphi(s)$. The theorem is proved.

The proof of the next theorem requires the following lemma.

(3. 18) LEMMA. Let $T$ be a superior extension of the partially ordered set $S$. Let $t_{1}, t_{2} \in T$. Then $t_{1}=t_{2}$ if and only if $L\left(S, t_{1}\right)=L\left(S, t_{2}\right)$.

Proof. Let $L_{i}=L\left(S, t_{i}\right) i=1,2$. If $t_{1}=t_{2}$, clearly $L_{1}=L_{2}$. Conversely, let $L_{1}=L_{2}$. Since $T$ is a superior extension of $S, t_{i}=\sup L_{i}$. Hence $t_{1}=\sup \cdot L_{1}=$ $=\sup L_{2}=t_{2}$.

(3. 19) THEOREM. Let $S$ be a subset of a partially ordered set $T$. Then $T$ is a superior extension of $S$ if and only if there exists an order isomorphism $\beta$ of $T$ into $\mathscr{I}(S)$ where $\beta \mid S=\iota$.

Proof. (i) Suppose $T$ is a superior extension of $S$. Define $\beta(t)=L(S, t)$. Since $t$ is a superior element, $\beta(t) \neq t \varnothing$, whence $\beta(t) \in \mathscr{I}(S)$ and if $t \in S$, then $\beta(t)=\iota(t)$. Obviously, $\beta$ is order preserving. Suppose $\beta\left(t_{1}\right) \subseteq \beta\left(t_{2}\right)$. Then $t_{1}$ is an upper bound for $\beta\left(t_{2}\right)$. Since $T$ is a superior extension of $S$, we have $t_{2}=\sup \beta\left(t_{2}\right)$, whence $t_{1} \geqq t_{2}$. By (3. 18) and (2. 15.4), $\beta$ is an order isomorphism.

(3. 20) THEOREM. Let $T$ be a superior completion of a partially ozdered set $S$. Then there exists an order preserving mapping $\alpha$ of $\mathscr{I}(S)$ onto $T_{+}$such that $\alpha \iota=\varepsilon \mid S$ and if $\mathscr{J} \subseteq \mathscr{I}(S)$, then $\alpha(\sup \mathscr{J})=\sup (\alpha(\mathscr{J}))$.

Proof. If $I \in \mathscr{I}(S)$, then $I \subseteq S \subseteq T$ and we define $\alpha(l)=\sup I$, where the supremum is taken in $T_{+}$, and hence exists by (3.9). Clearly $\alpha$ is order preserving and $\alpha \iota(s)=s$. Hence $\alpha(\sup \mathscr{J}) \geqq \sup \alpha(\mathscr{J})$, for all $\mathscr{J} \subseteq \mathscr{I}(S)$.

It remains to show that $\alpha(\sup \mathscr{J}) \leqq \sup \alpha(\mathscr{J})$, for all $\mathscr{J} \subseteq \mathscr{I}(S)$. Note that $\sup \mathscr{J}=\cup \mathscr{J}=I_{0}$, say, and $I_{0} \subseteq S$. Let $s \in J$, for some $J \in \mathscr{J}$. Hence $s \leqq \sup J=$ $=\alpha(J) \leqq \sup \alpha(\mathscr{J})$, whence sup $\alpha(\mathscr{J})$ is an upper bound for $I_{0}$. The theorem follows.

It is interesting to compare the above theorem with (2.27). In general $\alpha$ commutes with supremum only when $\alpha$ is order true, but the special nature of $\mathscr{I}(S)$ yields this commutativity even though the mapping $\alpha$ in the theorem will rarely be order true if $S$ is not linearly ordered.

The above theorem says that every superior completion is, in some sense, a homomorphic image of $\mathscr{I}(S)$. The preceding theorem says that every superior extension is essentially a subset of $\mathscr{I}(S)$. We see therefore that $\mathscr{I}(S)$ is the universal superior extension of $S$.

(3. 21) EXAMPLE. Let $S$ consist of three pairwise incomparable elements $a, b, c$. Then $S_{+}=\{a, b, c,+\infty\}$ and $\mathscr{I}(S)$ consists of the seven non-empty subsets of $\{a, b, c\}$ ordered by inclusion. Let $T=S_{+}$. If $\alpha$ is the mapping of (3.20) and $I \subseteq \mathscr{I}(S)$ where $|I| \geqq 2$, then $\alpha(I)=+\infty$. In $\mathscr{I}(S),\{a, b\} \|\{\{c\}$, but $\alpha(\{a, b\})=+\infty>c=$ $=\alpha(\{c\})$ in $T$. Hence $\alpha$ is not order true, by (2.15.1).

(3. 22) Definition. Let $S$ be a partially ordered set. By $\Im(S)$ we mean the collection of all non-empty initial segments of $S$ which are bounded above, viz., $\mathfrak{J}(S)=\{\mathfrak{I} \in \mathscr{I}(S): \exists s \in S, \mathfrak{I} \leqq \iota(s)\}$. Dually, $\quad F(S)=\{F \in \mathscr{F}(S): \exists s \in S, F \geqq \varphi(s)\}$. 
In certain situations one does not wish a completion to have an infinite maximal element, unless the original set does. In these situations it will be necessary to use $\mathfrak{I}(S)$ rather than $\mathscr{I}(S)$. For example, when completing the rationals, one does not always wish to adjoin $+\infty$.

(3. 23) Remarks. (1) Note that $\Im(S)$ is of the same type (cf. (2.40)) as $S$.

(2) Note that $\mathfrak{I}(S)$ is the initial segment generated by $\iota(S)$ in the partially ordered set $\mathscr{I}(S)$. Hence if $\mathscr{A} \subseteq \mathfrak{I}(S)$ is bounded above in $\mathfrak{I}(S)$, then the suprema of $\mathscr{A}$ in $\mathscr{I}(S)$ and $\mathfrak{I}(S)$ coincide.

(3. 24) LemMa: Let $S$ be a partially ordered set. Then $\mathfrak{I}(S)$ is a superior completion of $S$ under the embedding $\iota$.

Proof. Let $\mathscr{A}$ be a subset of $\Im(S)$ which is bounded above by $I \in I(S)$. Then there exists $s \in S$ such that $I \subseteq \iota(s)$. Hence $\cup \mathscr{A} \subseteq I \subseteq \iota(s)$, whence sup $\mathscr{A}=\cup \mathscr{A} \in \mathfrak{I}(S)$. Hence $\mathfrak{I}(S)$ is complete and since $\mathfrak{I}(S) \subseteq \mathscr{I}(S), \mathfrak{I}(S)$ is a superior completion of $S$ under $\iota$.

We end this section with a proposition that will be useful in $\S 5$.

If $B$ is a subset of the partially ordered set $A$, we shall now write $\sup _{A} B\left(\inf _{A} B\right)$ for the supremum (infimum) of $B$ in $A$.

(3. 25) Propositron. Let $T$ be a superior extension of a partially ordered set $S$. If $B \leqq S$ and $\inf _{S} B$ exists, then $\inf _{T} B$ exists and $\inf _{T} B=\inf _{S} B$.

Proof. Let $b=\inf _{s} B$, and let $t \in T$ be any lower. bound for $B$. If $s \in S$ and $s \leqq t$ then $s$ is a lower bound for $B$, whence $s \leqq b$. Thus $b$ is an upper bound for $L(S, t)$. Since $T$ is a superior extension of $S, t=\sup L(S, t)$, whence $t \leqq b$. Hence $b=\inf _{T} B$.

(3. 26) Corollary. Let $T$ be a normal extension of a partially ordered set $S$. If $B \subseteq S$ and $\sup _{S} B\left(\inf _{S} B\right)$ exists, then $\sup _{T} B,\left(\inf _{T} B\right)$ exists and $\sup _{T} B=\sup _{S} B$ $\left.\operatorname{(inf}_{T} \bar{B}=\inf _{S} B\right)$.

Proof. Immediate by $(3.25)$ and $(3.25)^{*}$.

\section{Abnormal completions of linearly ordered sets}

In this section we shall show that every linearly ordered set has a universal abnormal completion $\mathscr{F}(\mathscr{I}(S))=\mathscr{F} \mathscr{I}(S)$. Every abnormal extension of $S$ can be embedded order isomorphically in $\mathscr{F} \mathscr{I}(S)$ (cf. (4. 14)), and every abnormal completion of $S$ is essentially an order homomorphic image of $\mathscr{F} \mathscr{I}(S)$ (cf. (4.12)).

(4. 1) Notation. Let $S$ be a linearly ordered set. Elements of $\mathscr{F} \mathscr{I}(S)$ will be denoted by capital Greek letters. We denote by $\iota$ and $\iota^{-}$the mappings of $S$ into $\mathscr{I}(S) \cup\{\varnothing\}$ defined in (3.2), but $\varphi$ and $\varphi^{+}$will be the mappings of (3.2) with $S$ replaced by $\mathscr{I}(S)$. We define $\pi=\varphi \iota$, and $\pi^{+}=\varphi^{+} \iota$. Note that $\pi(s) \in \mathscr{F} \mathscr{I}(S)$ for $s \in S$ and $\pi^{+}(s) \in \mathscr{F} \mathscr{I}(S)$, for $s \in S$, unless $s$ is the maximal element of $S$. Next, put $\pi^{+-}(s)=\varphi^{+}{ }^{-}(s)$, unless $s$ is the minimal element of $S$, in which case $\iota^{-}(s) \notin \mathscr{I}(S)$; in this case we set $\pi^{+-}(s)=\mathscr{I}(S)$. Finally, we set $\pi^{-}(s)=\varphi \iota^{-}(s)$, if $s$ is not the minimal element of $S$, in which case $\pi^{-}(S)$ is undefined.

(4. 2) REMARKS. These remarks follow from (3.4). 
(1) If $s$ is not the maximal element in $S$, then in $\mathscr{F} \mathscr{I}(S), \pi(s)^{+}$exists and $\pi(s)^{+}=\pi^{+}(s)$. If $s$ is not the minimal element in $S$, then $\pi(s)^{-}=\pi^{-}(s)$. Thus, if $\Delta \in \pi(S)$, and $\Delta$ is neither maximal nor minimal in $\mathscr{F} \mathscr{I}(S)$, then $\Delta$ has both a predecessor and a successor in $\mathscr{F} \mathscr{I}(S)$. If $s^{+}$exists, then $\pi^{+}(s)=\pi\left(s^{+}\right)$and if $s^{-}$exists then $\pi^{-}(s)=\pi\left(s^{-}\right)$. The above mappings are 1-1, since in the linearly ordered case $\varphi, \iota, \varphi^{+}, \iota^{-}$are all $1-1$.

(2) If $\pi(s)^{+}=\pi(t)$, then $t=s^{+}$.

(3) If $\pi(s)^{-}=\pi(t)$, then $t=s^{-}$.

(4) $\pi^{+-}=\pi$.

(4. 3) Proposition. Let $S$ be a linearly ordered set. Then $\pi(S)=\varphi(\mathscr{I}(S)) \cap$ $\cap\left(\varphi^{+}(\mathscr{I}(S) \downarrow) \cup\{\mathscr{I}(S)\}\right)$, where $\mathscr{I}(S) \uparrow=\mathscr{I}(S) \backslash\{S\}$.

Proof. Let $s \in S$. Then $\pi(s)=\varphi \iota(s) \in \varphi(\mathscr{I}(S))$. If $s$ is the minimal element of $S$, then $\iota(s)=\{s\}$, whence $\pi(s)=\varphi(\{s\})=\mathscr{I}(S)$. On the other hand if $s$ is not the minimal element of $S$, then $\pi(s)=\varphi^{+} \iota^{-}(s) \in \varphi^{+}(\mathscr{I}(S) \downarrow)$, since $\iota^{-}(s) \neq S$. Thus $\pi(S) \subseteq \varphi(\mathscr{I}(S)) \cap\left(\varphi^{+}(\mathscr{I}(S) \downarrow) \cup\{\mathscr{I}(S)\}\right)$. Conversely, let $\Delta \in \varphi(\mathscr{I}(S)) \cap\left(\left\{\varphi^{+}(\mathscr{I}(S) \downarrow)\right.\right.$ $\cup\{\mathscr{I}(S)\})$. Thus $\Delta=\varphi(I)$, for some $I \in \mathscr{I}(S)$. If $\Delta=\mathscr{I}(S)$, then $I$ is the minimal element of $\mathscr{I}(S)$, whence $I=\iota(s)$, where $s$ is the minimal of $S$. Thus $\Delta \in \pi(S)$. If $\Delta \in \varphi^{+}(\mathscr{I}(S) \downarrow)$ say $\Delta=\varphi^{+}(J)$, then by $(3.4 .4)^{*}, I=J^{+}$. Since $S$ is linearly ordered and $I=J^{+}, I \backslash J=\{s\}$, for some $s \in S$. It is clear that $I=\imath(s)$, whence $\Delta=\varphi(s) \in \pi(S)$.

(4. 4) REMARK. If $\Delta, \Gamma \in \mathscr{F} \mathscr{F}(S)$, then by (3.3)*, $\Delta \leqq \Gamma$ if and only if $\Delta \supseteqq \Gamma$. Also, since $\mathscr{F} \mathscr{I}(S)$ is an inferior completion of $\mathscr{I}(S)$, and $\mathscr{I}(S)$ is linearly ordered by (3. 3), it follows by (3.3)* that $\mathscr{F} \mathscr{I}(S)$ is linearly ordered.

(4. 5) LemMa. If $\Delta \in \varphi(\mathscr{I}(S))$, then $\Delta$ is a superior element of $\pi(S)$ in $\mathscr{F} \mathscr{I}(S)$.

Proof. By hypothesis, $\Delta=\varphi(I)$, for some $I \in \mathscr{I}(S)$. We shall show that $\Delta=$ $=\sup \{\pi(s): s \in I\}=\sup \pi(I)$. By (3. 10)* (cf. discussion after (3. 14)) $\sup \pi(I)=\bigcap \pi(I)$ since $\pi(I)$ is bounded above by $\varphi(I)$. If $J \in \mathscr{I}(S)$, then $J \in \pi(s)$ if and only if $J \supseteq \iota(s)$. Hence, $J \in \cap \pi(I)$ if and only if $J \supseteqq \cup\{c(s): s \in I\}=\cup \iota(I)=I$. Hence sup $\pi(I)=$ $=\bigcap \pi(I)=p(I)$.

(4. 6) LEMMA. If $\Delta \in \varphi^{+}(\mathscr{I}(S) \downarrow) \cup\{\mathscr{I}(S)\}$, where $\mathscr{I}(S) \downarrow=\mathscr{I}(S) \backslash\{S\}$, then $\Delta$ is an inferior element of $\pi(S)$ in $\mathscr{F} \mathscr{I}(S)$.

Proof. If $\Delta=\varphi^{+}(I)$, for some $I \in \mathscr{I}(S)$, we define

(a) $\Gamma=\inf \{\pi(s): s \notin I\}=\inf \pi(S \backslash I)$.

If $\Delta=\mathscr{I}(S)$, we set $I=\varnothing$ and again define $\Gamma$ by (a). By (3. 10)*,$\quad \Gamma=\cup \pi(S \backslash I)$. If $J \in \Gamma$, there exists $s \notin I$, such that $J \in \pi(s)$. For this $s, J \supseteqq \iota(s)$ and since $S$ is linearly ordered and $s \notin I$, we have $\iota(s) \supset I$. Hence $J \supset I$. Conversely, if $J \in \mathscr{I}(S)$ and $J \supset I$, then there exists $s \in J \backslash I$ and for this $s$, both $s \notin I$ and $J \supseteqq \iota(s)$, whence $J \in \pi(s)$. It follows that $J \in \Gamma$. Thus $\Gamma=\{J \in \mathscr{I}(S): J \supset I\}$, and so if $\bar{I} \subseteq \mathscr{I}(S), \Gamma=\varphi^{+}(I)=\Delta$, and if $I=\varnothing, \Gamma=\mathscr{I}(S)=\Delta$.

(4. 7) Lemma. Let $S$ be a linearly ordered set. Then

$$
\mathscr{F} \mathscr{I}(S)=\varphi(\mathscr{I}(S)) \cup \varphi^{+}(\mathscr{I}(S) \downarrow) \cup\{\mathscr{I}(S)\},
$$

where $\mathscr{I}(S) \downarrow=\mathscr{I}(S) \backslash\{S\}$. Further if $\Delta \in \varphi(\mathscr{I}(S))$ then $\Delta^{+}$exists, and if $\Delta \in \varphi^{+}(\mathscr{I}(S) \downarrow)$ then $\Delta^{-}$exists. 
Proof. The first part of the lemma follows from (3.13), (2.9) and (3.16)*. Since $\mathscr{I}(S)$ is linearly ordered, $\varphi^{+}(I)=\varphi(I)^{+}$and $\varphi^{+}(I)^{-}=\varphi(I)$ by $(3.4$. 3)*

(4. 8) LEMMA. Let $S$ be a linearly ordered set. If $\Delta$ is a normal element of $\pi(S)$ in $\mathscr{P} \mathscr{I}(S)$, then $\Delta \in \pi(S)$.

Proof. Let $\Delta=\inf \mathscr{P}_{1}=\sup \mathscr{P}_{2}$, where $\mathscr{P}_{i} \subseteq \pi(S), i=1,2$. If $\Delta$ has either a successor or a predecessor, then $\Delta \in \mathscr{P}_{1}$ or $\Delta \in \mathscr{P}_{2}$, resp., whence $\Delta \in \pi(S)$. However, by (4.8) every $\Delta$, except possibly $\mathscr{I}(S)$, has a successor or predecessor. If $\Delta=\mathscr{I}(S)$, then $\Delta$ is the minimal element of $\mathscr{F} \mathscr{I}(S)$ whence $\mathscr{P}_{2}=\{d\}$. It follows that $\Delta \in \pi(S)$.

(4. 9) THEOREM. Let $S$ be a linearly ordered set. Then,

(1) $\mathscr{F} \mathscr{I}(S)$ is a linearly ordered abnormal completion of $S$ under the embedding $\pi$.

(2) $\mathscr{F} \mathscr{I}(S)=\varphi(\mathscr{I}(S)) \cup \varphi^{+}(\mathscr{I}(S) \downarrow) \cup\{\mathscr{I}(S)\}$, where $\mathscr{I}(S) \downarrow=\mathscr{I}(S) \backslash\{S\}$.

(3) $\Delta$ is a superior element of $\pi(S)$ in $\mathscr{F} \mathscr{I}(S)$ if and only if $\Delta \in \varphi(\mathscr{I}(S))$;

(4) $\Delta$ is an inferior element of $\pi(S)$ in $\mathscr{F} \mathscr{I}(S)$ if and only if $\Delta \in \varphi^{+}(\mathscr{I}(S) \downarrow) \cup$ $\cup\{\mathscr{I}(S)\}$.

(5) $\Delta$ is a normal element of $\pi(S)$ in $\mathscr{F} \mathscr{I}(S)$ if and only if $\Delta \in \pi(S)$.

Proof. By (3. 3), (3.3)* and (3. 10)*, $\mathscr{F} \mathscr{I}(S)$ is complete and linearly ordered, and (2) is merely (4.7). Let $\mathscr{U}$ be the set of superior elements and $\mathscr{L}$ be the set of inferior elements of $\pi(S)$ in $\mathscr{F} \mathscr{I}(S)$. By (4. 5),

and by (4.6)

$$
\mathscr{U} \supseteqq \varphi(\mathscr{I}(S))
$$

$$
\mathscr{L} \supseteqq \varphi^{+}(\mathscr{I}(S) \downarrow) \cup\{\mathscr{I}(S)\}
$$

whence by (2), $\mathscr{U} \cup \mathscr{L}=\mathscr{F} \mathscr{I}(S)$, and hence $\mathscr{F} \mathscr{I}(S)$ is an abnormal completion of $S$ under $\pi$. Thus (1) is established.

From (a), (b) and (4.8) it follows that

$$
\varphi(\mathscr{I}(S)) \cap\left(\varphi^{+}(\mathscr{I}(S) \downarrow) \cup\{\mathscr{I}(S)\}\right) \subseteq \mathscr{U} \cap \mathscr{L} \subseteq \pi(S) .
$$

But (4. 3) now yields

$$
\varphi(\mathscr{I}(S)) \cap\left(\varphi^{+}(\mathscr{I}(S) \downarrow) \cup\{\mathscr{I}(S)\}=\mathscr{U} \cap(\mathscr{L}=\pi S) .\right.
$$

By standard set theoretic arguments, we deduce from (2), (a), (b) and (c) that

$$
\left.\mathscr{U}=\varphi(\mathscr{I}(S)) \text { and } \mathscr{L}=\varphi^{+}(\mathscr{I}(S) \downarrow) \cup\{\mathscr{I}(S)\}\right) \text {. }
$$

The theorem is proved.

(4. 10) LEMMA. Let $T$ be an abnormal completion of a linearly ordered set $S$ under the identity. Let $S_{1}, S_{2}$ be subsets of $S$ such that $\sup \pi\left(S_{1}\right)=\sup \pi\left(S_{2}\right)$ $\left(\inf \pi\left(S_{1}\right)=\inf \pi\left(S_{2}\right)\right)$ in $\mathscr{F} \mathscr{I}(S)$. Then $\sup S_{1}=\sup S_{2} \quad\left(\inf S_{1}=\inf S_{2}\right)$ in $T$.

Proof. Let $\Delta=\sup \pi\left(S_{1}\right)=\sup \pi\left(S_{2}\right)$. If $\Delta=\pi(s)$, then since by (4.2.1) either $s$ is minimal in $S$ or $\pi^{-}(s)=\pi(s)^{-}<\pi(s)$, it follows that $s \in S_{i}, i=1,2$ whence $s$ is the maximal element of $S_{i}$. Hence $\sup \left(S_{1}\right)=s=\sup \left(S_{2}\right)$ in $T$.

Suppose now that $\Delta \& \pi(S)$, and so $S_{i}, i=1,2$, has no maximal element. Let $s \in S_{1}$. Since $\Delta=\sup \pi\left(S_{2}\right)$ and $\pi(s)<\Delta$, there exists $t \in S_{2}$ such that $\pi(s) \leqq \pi(t)$. 
But $\pi$ is an order isomorphism, and so $s \leqq t$. It follows that $\sup \left(S_{1}\right) \leqq \sup \left(S_{2}\right)$ in $T$. Interchanging indices, we obtain $\sup \left(S_{2}\right) \leqq \sup \left(S_{1}\right)$, and so $\sup \left(S_{1}\right)=\sup \left(S_{2}\right)$ in $T$. The dual argument works for infima.

(4. 11) THeOREM. Let $T$ be an abnormal completion of a linearly ordered set $S$ under the identity mapping. Then there exists a unique order preserving mapping $\alpha$ of $\mathscr{F} \mathscr{I}(S)$ onto $T_{ \pm}$such that

$$
\alpha \pi=\varepsilon \mid S .
$$

Further, this mapping $\alpha$ preserves suprema and infima; i.e. if $\mathscr{D} \subseteq \mathscr{F} \mathscr{I}(S)$, then $\alpha(\sup \mathscr{D})=\sup \alpha(\mathscr{D}),(\alpha(\inf \mathscr{D})=\inf \alpha(\mathscr{D}))$.

Proof. For $\pi(s) \in \pi(S)$, we define $\alpha \pi(s)=s$. Let $\Delta \in \mathscr{F} \mathscr{F}(S) \backslash \pi(S)$. By (4.9) then either there exists $\mathscr{P} \subseteq \pi(S)$ such that either $\Delta=$ sup $\mathscr{P}$ or there exists $\mathscr{P} \subseteq \pi(S)$ such that $\Delta=\inf \mathscr{P}$, but not both. We may now define $\alpha(\Delta)=\sup \alpha(\mathscr{P})$ or $\alpha(\Delta)=$ $=\inf \alpha(\mathscr{P})$, respectively. By $(4,10), \alpha$ is well-defined.

To show that $\alpha$ is order preserving, let $\Delta_{1}>\Delta_{2}$. There are four possible cases, according as $\Delta_{1}$ and $\Delta_{2}$ are superior or inferior elements. We consider here only the most difficult case in which $\Delta_{1}$ is a superior and $\Delta_{2}$ is an inferior element. Let $\Delta_{1}=\sup \mathscr{P}_{1}$, and $\Delta_{2}=\inf \mathscr{P}_{2}$, where $\mathscr{P}_{i} \subseteq \pi(S)$. Since $\Delta_{2}<\Delta_{1}=\sup \mathscr{P}_{1}$, there exists $\Gamma_{1} \in P_{1}$ with $\Delta_{2}<\Gamma_{1} \leqq \Delta_{1}$. Since $\Gamma_{1}>A_{2}=\inf \mathscr{P}_{2}$, there exists $\Gamma_{2} \in \mathscr{P}_{2}$ with $\Delta_{2} \leqq \Gamma_{2}<\Gamma_{1} \leqq \Delta_{1}$. Since $\alpha$ is an order isomorphism on $\pi(S)$, we obtain

$$
\alpha\left(\Delta_{2}\right)=\inf \alpha\left(\mathscr{P}_{2}\right) \leqq \alpha\left(\Gamma_{2}\right) \leqq \alpha\left(\Gamma_{1}\right) \leqq \sup \alpha\left(\mathscr{P}_{1}\right)=\alpha\left(\Delta_{1}\right)
$$

Thus, in this case, $\alpha$ is order preserving.

We next show that $\alpha$ is onto $T_{ \pm}$. Let $t \in T_{ \pm}$. Since $T_{ \pm}$is an abnormal extension of $S$, either $t \in S$ or $t=\sup L^{\#}(S, t)=\sup L^{\#}$ or $t=\inf U^{\#}(S, t)=\inf U^{\#}$. If $t \in S$, then $t=\alpha \pi(t)$. If $t \notin S$, suppose $t=\sup L^{\#}$, and let $\Delta=\sup \pi\left(L^{\#}\right)$. Since $L^{\#}$ has no maximal element, and since $\pi$ is an order isomorphysm, it follows that $\Delta$ is not the minimal element of $\mathscr{F} \mathscr{I}(S)$ and $\Delta$ has no predecessor. Hence by (4. 3. 1) $\Delta \notin \pi(S)$, Thus by definition of $\alpha, \alpha(\Delta)=\sup \{\alpha \pi(s): s<t\}=\sup L^{\#}=t$. The case in which $t$ is an infimum follows similarly. The uniqueness of $\alpha$ is a consequence of (2.30). It follows from (2.27) that $\alpha$ commutes with the supremum and infimum operations.

(4. 12) REMARK. Let $S$ be a linearly ordered set. We note that $\Im(S) \subseteq \mathscr{I}(S)$, but that $\mathfrak{F} \Im(S)$ is not a subset of $\mathscr{F} \mathscr{I}(S)$, if $S$ has no maximal element. If $\Gamma \in \mathcal{F} \mathfrak{I}(S)$, then $\Gamma \leqq \Im(S) \leqq \mathscr{I}(S)$, and let $\eta \Gamma$ be the smallest final segment of $\mathscr{I}(S)$ containing $\Gamma$. Clearly $\eta \Gamma=\Gamma$. or $\eta \Gamma=\Gamma \backslash\{S\}$, according as $S$ has a maximal element or not. Also $\mathscr{F} \mathscr{I}(S)=\eta(\mathscr{F} \mathfrak{I}(S)) \cup\{\mathscr{I}(S),\{S\}\}$ where $\{S\}=+\infty$ and $\mathscr{I}(S)=-\infty$. Obviously $\eta$ is $1-1$. For the sake of clearity we omit writing $\eta$ where convenient. Thus in (4. 13) below we write $\pi$ where more precisely we mean $\eta^{-1} \pi$.

(4.13) COROLLARY. Let $T$ be an abnormal completion of a linearly ordered set $S$ of the same type as $S$. Then there exists a unique order preserving mapping $\alpha$ of $\mathfrak{F} \Im(S)$ onto $T$ such that $\alpha \pi=\varepsilon \mid S$. The mapping $\alpha$ satisfes the additional conditions of (4. 11).

Proof. If $S=S_{ \pm}$, then $T=T_{ \pm}$, and the corollary reduces to Theorem (4. 11). We argue the case in which $S$ has a minimal but no maximal element. Then $S_{+}=S_{ \pm}$, and $T_{ \pm}=T_{+}=T \cup(+\infty)$, and $\mathscr{F} \mathscr{I}(S)=\mathscr{F} \mathfrak{I}(S) \cup\{\{S\}\}$. By (4.11), there exists 
P.

an order preserving mapping $\alpha^{\prime}$ of $\mathfrak{F} \Im(S) \cup\{\{S\}\}$ into $T \cup\{+\infty\}$ such that $\alpha^{\prime} \pi=\varepsilon \mid S$. Obviously $\alpha^{\prime}(\{S\})=+\infty$, and hence if $\alpha=\alpha^{\prime} \mid \mathfrak{F} \Im(S)$, then $\alpha$ is into $T$ and $\alpha \pi=\varepsilon \mid S$. - The other cases follow similar]y.

(4. 14) THEOREM. Let $T$ be an abnormal extension of a linearly ordered set $S$. Then $T$ can be embedded order isomorphically into $\mathscr{F} \mathscr{H}(S)$ in such a fashion that $s$ maps onto $\pi(s)$, for each $s \in S$. Further if $\beta$ is a mapping of $T$ into $\mathscr{F} \mathscr{F}(S)$ then the following are equivalent: in (4. 11)

(i) $\alpha \beta=\varepsilon \mid T$ where $\alpha$ is the unique mapping of $\mathscr{F} \mathscr{F}(S)$ onto $T_{ \pm}$determined

(ii) $\beta$ is order isomorphism of $T$ into $\mathscr{F} \mathscr{I}(S)$ such that $\beta \mid S=\pi$.

Proof. By (4. 11) there exists a mapping $\alpha$ of $\mathscr{F} \mathscr{I}(S)$ onto $T_{ \pm}$which satisfies the hypothesis of (2.47) with $Z=\mathscr{F} \mathscr{I}(S), X=\pi(S)$, and $Y=T_{ \pm}$. Thus by (2. 47), there exists a mapping $\beta^{\prime}$ which embeds $T_{ \pm}$order isomorphically into $\mathscr{F} \mathscr{I}(S)$ such that $\beta^{\prime} \alpha|\pi(S)=\varepsilon| \pi(S)$. Thus setting $\beta=\beta^{\prime} \mid T$, we obtain $\beta(s)=\beta \alpha \pi(s)$, for $s \in S$. The equivalence of (i) and (ii) also follows from (2.47).

(4.15) COROLlary. Let $T$ be be an abnormal extension of a linearly ordered set $S$ of the same type as $S$. Then $T$ can be emoedded order isomorphically into $\mathfrak{F}(S)$ such that $s$ maps onto $\pi(s)$, for each $s \in S$. The rest of $(4.14)$ holds with $\mathscr{F} \mathscr{I}(S)$ replaced by $\mathfrak{F}(S)$.

Proof. Similar to (4. 13).

(4. 16) COROLlaRy. The number of ways that a given extension $T$ of $S$ can be embedded in $\mathscr{F} \mathscr{I}(S)$ so that $s$ maps onto $\pi(s)$, for all $s \in S$ is at most $2^{2\left(2^{|s|}-1\right)}$.

Proof. By (2. 48. 2) we obtain the bound $2^{|T| S \mid}$. But by (2. 49), $|T| \leqq 2\left(2^{|S|}-1\right)$.

(4. 17) REMARK. This bound can be attained, e.g., let $S=\mathbf{Q}$, the rationals, and let $T=\mathbf{R}$, the reals.

(4. 18) Proposition. Let $S$ be a linearly ordered set. Then the number of $a b$ normal extensions of $S$ which are distinct under order isomorphisms leaving $S$ pointwise fixed is at most $2^{2|s|}$.

Proof. If $S$ is finite, then $S$ is the only abnormal extension of $S$. If $S$ is infinite $|\mathscr{F} \mathscr{I}(\dot{S})| \leqq 2^{|S|}$, by (2.49). By (4.14) each abnormal extension of $S$ is order isomorphic to a subset of $\mathscr{F} \mathscr{I}(S)$ which contains $S$. The number of all subsets of $\mathscr{F} \mathscr{I}(S)$ is $2^{2|S|}$, and the result follows.

(4. 19) REMARK. By taking $S=\mathbf{Q}$, we obtain a situation in which the number of abnormal extensions equals $2^{2^{|S|}}=2^{\mathfrak{c}}$, where $\mathrm{c}$ is the power of the continuum.

(4. 20) THEOREM. Let $T$ be a lineurly ordered extension of $S=S_{ \pm}$. Then $T$ is an abnormal completion of $S$ if and only if there exists an order preserving mapping $\alpha$ of $\mathscr{F} \mathscr{F}(S)$ onto $T$ such that $\alpha \pi=\varepsilon \mid S$.

Proof. Theorem (4.11) guarantees the existence of $\alpha$ in the case, when $T$ is an abnormal completion, since here $T=T_{ \pm}$.

Suppose now there exists such a mapping $\alpha$. Then, by (2.27) and the fact that $\mathscr{F} \mathscr{I}(S)$ is complete, it follows that $T$ is complete. By (2.28), $T$ is an abnormal completion of $S$. 
(4.21) Corollary. Let $T$ be a linearly ordered extension of $S$ of the same type as $S$. Then $T$ is an abnormal completion of $S$ if and only if there exists an order preserving mapping $\alpha$ of $\mathfrak{F} \Im(S)$ onto $T$ such that $\alpha \pi=\varepsilon \mid S$.

Proof. Compare (4.13) and (4.15). by $\pi^{*}$.

The set $\mathscr{F} \mathscr{I}(S)$ has a dual, viz. $\mathscr{I} \mathscr{F}(S)$. The mapping dual to $\pi$ will be denoted

(4. 22) Proposition. Let $S$ be any linearly ordered set. $\alpha \pi=\pi^{*}$.

(1) There exists an order isomorphism $\alpha$ from $\mathscr{F} \mathscr{I}(S)$ onto $\mathscr{I} \mathscr{F}(S)$ such that

(2) $\alpha \mid \mathfrak{F} \mathfrak{J}(S)$ is an order isomorphism onto $\mathfrak{I} \mathfrak{F}(S)$.

Proof. (1) By (4.9)* $\mathscr{I \mathscr { F }}(S)$ is an abnormal extension of $S$. By (4. 14) there exists an order isomorphism $\alpha$ embedding $\mathscr{I} \mathscr{F}(S)$ into $\mathscr{F} \mathscr{I}(S)$ such that $\alpha \pi^{*}=\pi$. Dually, by (4. 14)*, we may embed $\mathscr{F} \mathscr{I}(S)$ into $\mathscr{I} \mathscr{F}(S)$ by a mapping $\alpha$ for which $\alpha \pi=\pi^{*}$. Thus $\alpha \alpha^{*}|\pi(S)=\varepsilon| \pi(S)$, whence by (2. 30) $\alpha \alpha^{*}=\varepsilon \mid \mathscr{F} \mathscr{I}(S)$, hence $\alpha$ is an isomorphism onto, and (1) follows.

(2) Under $\alpha$, the maximal (minimal) elements of $\mathscr{F} \mathscr{I}(S)$ go onto the maximal (minimal) element of $\mathscr{I} \mathscr{F}(S)$. Thus (2) follows.

We now consider normal completions of linearly ordered sets. We show that there is essentially only one normal completion for such a set. The reader may wish to refer back to definition (2.19) and (2.32).

(4. 23) THEOREM. Let $S=S_{ \pm}$be a linearly ordered set, and let $Z=\mathscr{F} \mathscr{I}(S)$. Then

(1) $\hat{Z}(\pi(S))=\hat{Z}$ is a normal completion of $S$ under $\gamma \pi$, where $\gamma$ is the natural mapping of $Z$ onto $Z$.

(2) If $T$ is a normal completion of $S$ under $\tau$, then $T$ is order isomorphic to $\hat{Z}$ under a mapping $\beta$ such that $\beta \tau=\gamma \pi$.

Proof. (1) From (2.34) and (4. 20) (with $T$ replaced by $\hat{Z}$ and $\alpha$ replaced by $\gamma$ ), it follows that $\hat{Z}$ is an abnormal completion of $S$ under $\gamma \pi$. Since $S=S_{ \pm}, \hat{Z}$ is of the same type as $S$, whence $\hat{Z}$ is normal by (2.42).

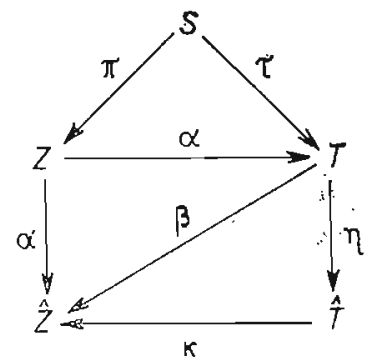

(2) The remainder of the proof consists in showing that the above commutative diagram exists and, $\eta$ and $\psi$ are isomorphisms. The existence of an order true map $\alpha$ onto $T$ such that $\alpha \pi=\tau$ follows from (4.20) (with $\varepsilon$ replaced by $\tau$ ). The existence of an order true map $\beta$ onto $Z$ such that $\beta \alpha=\gamma$, follows from (2.35). By (2.39), the natural mapping $\eta$ from $T$ onto $\hat{T}$ is an isomorphism. By (2.36) there is an iso- 
morphism $x$ of $\hat{T}$ onto $\hat{Z}$ such that $\beta=\eta$. Hence $\beta$ is also an isomorphism. The theorem is proved.

(4. 24) Corollary. Let $S$ be a linearly ordered set, and let $Z=\mathfrak{F} \mathfrak{I}(S)$. Then $\hat{Z}(\pi(S))=\hat{Z}$ is the unique normal completion of $S$. A set $T$ is a normal completion of $S$ under $\tau$ if and only if $T$ is order isomorphic to $\hat{Z}$ under a mapping $\beta$ such that $\beta \tau=\gamma \pi$, where $\gamma$ is the natural mapping of $Z$ onto $\hat{Z}$.

Proof. As for corollaries (4.13), (4.15) and (4.21) above. No restriction on $T$ is mecessary, since a normal completion $T$ of $S$ has the same type as $S$.

(4. 25) THEOREM. Let $T$ be an abnormal completion of a linearly ordered set $S$. Then $T$ is the (essentially) unique normal completion of $S$ if and only if the natural map $\eta$ of $T$ onto $\hat{T}$ is an order isomorphism and $T$ is of the same type as $S$.

Proof. By (4. 24) a normal completion is unique. By hypothesis, $T$ is a completion. Hence (2.41) proves the theorem.

(4. 26) COROLlary. Let $S$ be a linearly ordered set. Then $\widehat{\mathfrak{J}(S)}, \widehat{\mathfrak{F}(S)}, \widehat{\mathfrak{F} \Im(S)}$, and $\widehat{\mathfrak{F}}(S)$ are all isomorphic to the unique normal completion of $S$.

Proof. Immediate from (2.38) and (3.23.1).

\section{Partially ordered universal algebras}

(5. 1) Definition. A triple $\left({ }^{\prime}, \Omega, S\right)$ is a universal algebra if for each $\omega$ there is a (possibly infinite) cardinal number $n=n(\omega)$, such that ' $\omega$ is a function of $S^{n}$ into $S$. By convention $S^{0}=\{\varnothing\}$, and $n$ is identified with the least ordinal of cardinality $n$. Normally each $\omega \in \Omega$ is a function on $S^{n}$, and 'is the identity function, and bence not written. Because of the nature of our work, it is sometimes important to distinguish between $\omega$ and ' $\omega$, since we shall have several algebras with the same set $\Omega$ on the same set $S$.

(5.2) Notations AND DEFinition. If $\omega \in \Omega$ is $n$-ary, and $\mathbf{x} \in S^{n}$, we may write $\omega\left(x_{0}, x_{1}, \ldots\right)$ for $\omega(\mathbf{x})$. If $\eta$ is a function defined on a set $W$, and $\mathbf{x} \in W^{n}$, then we define $\eta(\mathbf{x})_{i}=\eta\left(x_{i}\right)$, all $i<n$. In particular this notation will frequently be used with $\eta=\sup , \iota, L(V):$, thus

$$
(\sup \mathbf{A})=\left(\sup A_{0}, \sup A_{1}, \ldots\right), \quad L(V, \mathbf{t})=\left(L\left(V, t_{0}\right), L\left(V, t_{1}\right), \ldots\right) .
$$

If $W$ is partially ordered, then $W^{n}$ is partially ordered coordinatewise by $\mathbf{x} \leqq \mathbf{y}$ if and only if $x_{i} \leqq y_{i}$, for all $i<n$.

(5.3) Definition. Let $\left({ }^{\prime}, \Omega, A\right)$ and $\left({ }^{\prime \prime}, \Omega, B\right)$ be universal algebras, and let $\eta$ be a mapping of $A$ into $B$. Then $\eta$ is a homomorphism from $\left(^{\prime}, \Omega, A\right)$ into $\left({ }^{\prime \prime}, \Omega, B\right)$ if and only if for all $\omega \in \Omega$, and all $\mathbf{x} \in A^{n}$ we have

$$
\eta\left({ }^{\prime} \omega(\mathbf{x})\right)=" \omega(\eta(\mathbf{x}))
$$

Various. results on universal algebras can be found in KUROSH [5] $\S 17$ and $\S 23$, and COHN [2], and will be assumed. 
(5.4) Notation. We denote the free universal algebra (Kurosh [5] §23, CoHN [2], Chapter III) generated by $X$ and $\Omega$ by $\langle\Omega, X\rangle$. For precise definitions which are also valid in the case of infinitary operations, see $\S 7$.

(5. 5) Definition. Let $S$ be a partially ordered set. We say that the universal algebra $(\Omega, S)$ is a partially ordered universal algebra if all $\omega \in \Omega$ are order preserving as mappings from $S^{n}$, partially ordered coordinatewise, to $S$, viz., $\mathbf{x} \leqq \mathbf{y}$ implies $\omega(\mathbf{x}) \leqq \omega(\mathbf{y})$.

(5.6) Definitions. Let $(\Omega, S)$ be a partially ordered universal algebra. Let $T$ be an extension of $S$ under the identity.

(1) A partially ordered universal algebra $\left({ }^{\prime}, \Omega, T\right)$ is an extension of $(\Omega, S)$ if for all $\mathbf{s} \in S^{n} \quad \omega(\mathbf{s})=\omega(\mathbf{s})$.

(2) The algebra $\left(-, \Omega, 2^{S}\right)$ is defined by $\bar{\omega}(\mathbf{A})=\left\{\omega(\mathbf{a}): a_{i} \in A_{i}\right.$, for all $\left.\left.i<n\right)\right\}$ for $\mathbf{A} \in\left(2^{S}\right)^{n}$ and $\omega$-ary.

(3) Let $\mathbf{t} \in T^{n}$. We define $* \omega(t)=\sup \bar{\omega}(L(S, \mathbf{t}))$ if this supremum exists.

(4) If for all $\omega \in \Omega, * \omega$ is defined everywhere, the $(*, \Omega, T)$ is an universal algebra. The algebra $(*, \Omega, T)$ obtained by this method of defining the operations on $T$ is called the superior extension of $(\Omega, S)$ to $T$. In general, there will be other extensions (', $\Omega, T)$ of $(\Omega, S)$.

(5) Let $(\Omega, S)$ be a partially ordered universal algebra, and let $\varrho$ be an order isomorphism of $S$ onto $\varrho(S)$. If $\omega \in \Omega$ is $n$-ary, and $\mathbf{s} \in S^{n}$ we define ' $\omega(\varrho(\mathbf{s}))=\varrho(\omega(s))$. Evidently (', $\Omega, \varrho(S))$ is isomorphic to $(\Omega, S)$. We often drop the prime.

(6) If $T$ is a superior completion of $S$ (under $\varrho$ ) and the extension (*, $\Omega, T$ ) of $(\Omega, \varrho(S))$ exists, then we shall call (*, $\Omega, T$ ) a superior completion of $(\Omega, S)$ (under $\varrho$ ).

(5.7) REMARK. If $T=T_{+}$is a superior completion of $S$ then * $\omega$ will be everywhere defined, since, as we recall, $T=T_{+}$if and only if every subset of $T$ has a supremum. Thus, in this case, $(*, \Omega, T)$ exists.

(5.8) Proposition. Let $(\Omega, S)$ be a partially ordered universal algebra. Let $T$ be a superior extension of $S$ such that $(*, \Omega, T)$ exists. Then $\left(^{*}, \Omega, T\right)$ is a partially ordered universal algebra into which $(\Omega, S)$ is embedded isomorphically in an order true fashion by the identity mapping.

Proof. It is easily verfied that $(*, \Omega, T)$ is a partially ordered universal algebra. It is also obvious that the identity mapping is $1-1$ and order true. So we need only show that $\varepsilon \mid S$ is a homomorphism, i.e., that $\omega(\mathbf{s})={ }^{*} \omega(\mathbf{s})$, for all $\mathbf{s} \in S^{\prime \prime}$. But this follows since $\omega$ is order preserving and $s_{i} \in I,\left(s_{i}\right)$, all $i<n$.

(5. 9) LEMMA. Let $(\Omega, B)$ be a partially ordered universal algebra. Let $A_{i} \subseteq B$ and let $a_{i}$ be an upper bound for the subset $A_{i}$ of $B, i<n$. Then, if $\omega \in \Omega$ is $n$-ary, $\omega(\mathbf{a})$ is an upper bound for $\bar{\omega}(\mathbf{A})$.

Proof. Follows immediately, since $\omega$ is order preserving.

(5.10) REMARK. There are examples of linearly ordered semigroups containing subsets $A_{1}, A_{2}$ for which $\sup A_{1}, \sup A_{2}$ and $\sup A_{1} A_{2}$ exist but $\sup A_{1} A_{2}<$ $<\left(\sup A_{1}\right)\left(\sup A_{2}\right)$. For example, let $A=[0,1]$ with $x y=0$ unless $x=y=1$, and $1 \cdot 1=1$. Let $A_{1}=A_{2}=[0,1)$.

We are therefore led to the following definition: 
(5. 11) Definition. Let $(\Omega, B)$ be a partially ordered universal algebra. We call $\omega \in \Omega, \omega n$-ary, suprema preserving if and only if for all $A_{i} \subseteq B, i<n$ such that $\sup A_{i}=$ $=a_{i}$ exists, we have $\omega(\mathbf{a})=\sup \bar{\omega}(\mathbf{A})$. We say that $(\Omega, B)$ is suprema preserving if all $\omega \in \Omega$ are suprema preserving.

(5. 12) REMARK. Let $(\Omega, S)$ be a partially ordered universal algebra. Since $\iota$ is a $1-1$ mapping of $S$ into $\mathscr{I}(S)=\mathscr{I}(S)_{\dot{+}},(5.6 .4)$ defines $(\Omega, \iota(S))$, a universal algebra isomorphic to $(\Omega, S)$. The superior completion $\mathscr{I}(S)$ of $\iota(S)$ yields the universal algebra $(*, \Omega, \mathscr{J}(S))$, in accordance with definition (5.6). In fact, in this algebra, if $\omega$ is $n$-ary and $\mathbf{A} \in \mathscr{F}(S)^{n}$, then $* \omega(\mathbf{A})$ is the initial segment generated by $\left\{\omega(\mathbf{a}): a_{i} \in A_{i}\right\}=\bar{\omega}(\mathbf{A})$.

(5.13) THEOREM. Every partially ordered universal algebra has a superior completion which is suprema preserving, viz., $(*, \Omega, \mathscr{I}(S))$ is such a completion of $(\Omega, S)$.

Proof. Since the ordering in $\mathscr{F}(S)$ is set inclusion, it follows from lemma (5.9) that it is sufficient to prove that

$$
* \omega(\sup \mathscr{A}) \leqq \sup { }^{*} \omega(\mathscr{A})
$$

for all subsets $\mathscr{A}_{i} \subseteq \mathscr{I}(S), i<n$. By definition of $* \omega$

$$
* \omega(\sup \mathscr{A})=\sup \bar{\omega}(L(\iota(S), \sup \mathscr{A})) \text {. }
$$

Note that if $\iota(s) \subseteq \sup \mathscr{B}=\iota \mathscr{B}$ then there exist $B \subseteq \mathscr{B}$ such that $\iota(s) \subseteq B$, whence

$$
\sup \bar{\omega}(L(\iota S), \sup \mathscr{A}) \leqq \sup \left\{* \omega(A): A_{i} \in \mathscr{A}_{i}\right\}=\sup * \omega(\mathscr{A}) \text {. }
$$

If $\left(^{*}, \Omega, T\right)$ exists, it is the minimal extension of $(\Omega, S)$ to $T$ in a natural partial ordering of extensions. To make this notion precise, we require a definition.

(5.14) Defintion. Let $(\Omega, A)$ and $\left(^{\prime}, \Omega, A\right)$ be two partially ordered universal algebras on $A$. We say that $(\Omega, A) \leqq\left(^{\prime}, \Omega, A\right)$ if and only if $\omega(a) \leqq{ }^{\prime} \omega(a)$, for all $\mathbf{a} \in A^{n}$ and all $\omega \in \Omega$.

(5. 15) Proposition. Let $(\Omega, S)$ be a partially ordered universal algebra and let $T$ be a superior extension of $S$. Let $\left.{ }^{\prime}, \Omega, T\right)$ be a partially ordered extension of $(\Omega, S)$.

(1) If $\left({ }^{\prime}, \Omega, T\right)$ preserves suprema then $(*, \Omega, T)$ exists as constructed in $(5,6)$ and $(', \Omega, T)=(*, \Omega, T)$.

(2) If $(*, \Omega, T)$ exists then $\left.{ }^{*}, \Omega, T\right) \leqq(', \Omega, T)$.

Proof. (1) Let $\omega \in \Omega$ and suppose ' $\omega$ preserves suprema. Then for $\mathbf{t} \in T^{n}$, ' $\omega(\mathbf{t})=$ $=\sup \bar{\omega}(L(S, \mathbf{t}))={ }^{*} \omega(\mathbf{t})$ whence ${ }^{*} \omega$ exists and ${ }^{*} \omega={ }^{\prime} \omega$.

(2) Let $\omega \in \Omega$ and suppose ${ }^{*} \omega$ is defined. Then ' $\omega(\mathbf{t}) \geqq \sup \bar{\omega}(L(S, \mathbf{t}))={ }^{*} \omega(\mathbf{t})$, by (5.9) and the result follows.

(5.16) Proposition. Let $(\Omega, S)$ be a partially ordered universal algebra, and let $(*, \Omega, T)$ be a superior extension of $(\Omega, S)$. If $(*, \Omega, T)$ preserves infima, then so does every extension (', $\Omega, T)$ of $(\Omega, S)$ to $T$.

Proof. Let $\omega \in \Omega$ and let $\mathbf{A} \in\left(2^{T}\right)^{n}$ such that $\inf _{T} A_{i}=a_{i}$ for all $i<n$. By (5.9) and (5.15.2) and since $(*, \Omega, T)$ preserves infima, inf $\frac{i}{\omega}(\mathbf{A})={ }^{*} \omega(\mathbf{a}) \leqq \omega^{\prime}(\mathbf{a}) \leqq$ $\leqq \inf _{T} \bar{\omega}(\mathbf{A})$ and ' $\omega(\mathbf{a})=\inf _{T} \bar{\omega}(\mathbf{A})$ follows. 
(5.17) LemMA. Let $(\Omega, S)$ be a partially ordered universal algebra and let $T$ be a superior extension of $S$. Let (', $\Omega, T)$ be a partially ordered extension of $(\Omega, S)$. If (', $\Omega, T)$ preserves infima, so does $(\Omega, S)$.

Proof. Let $\omega \in \Omega$ and let $A \in\left(2^{S}\right)^{n}$, such that $a_{i}=\inf _{S} A_{i}$. By (3.25) $a_{i}=\inf _{T} A_{i}$, and as $\left({ }^{\prime}, \Omega, T\right)$ is infima preserving, $\omega(\mathbf{a})={ }^{\prime} \omega(\mathbf{a})=\inf _{T} \bar{\omega}(\mathbf{A})=\inf _{S} \bar{\omega}(\mathbf{A})$, by (3.25). The result follows.

(5.18) REMARK. A dual theory exists for inferior extensions. Thus let $(\Omega, S)$ be a partially ordered universal algebra and let $T$ be an inferior extension of $S$. Let $\omega \in(\Omega, S)$ be $n$-ary. We define

$$
{ }_{*} \omega(\mathbf{t})=\inf \bar{\omega}(U(S, t))
$$

if the infimum exists for all $t \in T^{n}$. If ${ }_{*} \omega$ is defined for all $\omega \in(\Omega, S)$, we call the res ulting extension $\left(_{*}, \Omega, T\right)$ the inferior extension of $(\Omega, S)$ to $T$.

(5.19) Theorem. Let $(\Omega, S)$ be a partially ordered universal algebra. Let $N$ be a normal extension of $S$. If there exists a partially ordered extension $\left({ }^{\prime}, \Omega, N\right)$ of $(\Omega, S)$ which preserves both suprema and infima then

(1) (', $\Omega, N)$ is the unique partially ordered extension of $(\Omega, S)$ to $N$,

(2) $(\Omega, S)$ preserves both suprema and infima.

Proof. (1) By (5. 15.1) $(*, \Omega, N)$ exists and (', $\Omega, N)=\left(^{*}, \Omega, N\right)$ and by (5. 13. 1)* $\left.{ }_{*}, \Omega, N\right)$ exists and $\left(^{\prime}, \Omega, N\right)=\left({ }_{*}, \Omega, N\right)$. Hence $(*, \Omega, N)=\left({ }_{*}, \Omega, N\right)$. Hence by (5.15.2) (', $\Omega, N)$ is both the minimal and maximal extension of $(\Omega, S)$ in the ordering of (5.14). Hence $\left({ }^{\prime}, \Omega, N\right)$ is the unique extension of $(\Omega, S)$ to $N$.

(2) This follows immadiately from $(5.17)$ and $(5.17)^{*}$.

(5.20) THEOREM. Let $(\Omega, S)$ be a partially ordered universal algebra and let $N$ be a normal extension of $S$. If $(*, \Omega, N)$ exists and preserves infima, then $(*, \Omega, N)$ is the unique extension of $(\Omega, S)$ to $N$.

Proof. By $(5.15 .1)^{*},(*, \Omega, N)$ exists and $(*, \Omega, N)=(*, \Omega, N)$. Hence by (5. 15.2) and $(5.15 .2)^{*},(*, \Omega, N)$ is both maximal and the minimal extension of $(\Omega, S)$ to $N$. The result follows.

(5. 21) EXAMPLE. For partially ordered sets $S$, the converse of (5. 19) is false. Let $\mathbf{R}_{0}$ be the reals with 0 removed, and let $\mathbf{S}=\left(\mathbf{R}_{0}, 1\right) \cup\left(\mathbf{R}_{0}, 2\right)$ ordered thus: $(x, i) \leqq(y, j)$ if and only if $x<0$ and $y>0$ or $i=j$ and $x \leqq y$. Let $N=S \cup\{0\}$, where $0 \leqq(x, i)$ if and only if $x>0$ and $0 \geqq(x, i)$ if and only if $x<0$. Let $\Omega=\{\omega\}$, where $\omega$ is unary. Let $\omega(x, 1)=(x, 1)$, and for $x>0$, let $\omega(x, 2)=(x+1,2)$, while $x<0$, $\omega(x, 2)=(x-1,2)$. Then $(\Omega, S)$ preserves infima and suprema, and the unique extension ' $\omega$ of $\omega$ to $N$ yields ' $\omega(0)=0$. It is easy to see that $\left({ }^{*}, \Omega, N\right)=\left({ }_{*}, \Omega, N\right)=$ $=\left(^{\prime}, \Omega, N\right)$, but $(*, \Omega, N)$ does not preserve infima or suprema.

In $\S 6$ we shall prove that the converse of $(5.19)$ holds for linearly orderes sets.

We now investigate the extent to which identities and inequalities are preserved when a partially ordered universal algebra is extended.

(5. 22) Definition. Let $\langle\Omega, X\rangle$ be a free universal algebra, let $(\Omega, A)$ be a universal algebra. A function $\psi$ from $X$ to $A$ will be called a substitution. We extend the domain of $\psi$ to $\langle\Omega, X\rangle$ thus: if $w$ is a word in $\langle\Omega, X\rangle$ then we denote by $\psi^{\prime}(w)$ the element of $A$ obtained by substituting $\psi(x)$ for each occurrence of $x$ in $w$. 
We note that $\psi^{\prime}$ is thus a homomorphism of $\langle\Omega, X\rangle$ into $(\Omega, A)$, and every homomorphism can be realized in this fashion. Where no confusion should arise, we shall write $\psi$ in place of $\psi^{\prime}$.

(5.23) Definition. Let $(\Omega, S)$ be a partially ordered universal algebra, and let $(*, \Omega, T)$ be a superior completion of $(\Omega, S)$. If $\psi$ is a substitution of $X$ into $T$, then we define the mapping $\psi$ of $\langle\Omega, X\rangle$ into $T$ as follows: $\psi(w)=\sup \{\theta(w)$ : where $\theta$ is a substitution from $X$ to $S$ and $\theta(x) \in L(S, \psi(x))\}$ where the supremum exists by (5.9).

(5.24) ReMARK. We note that, in general, $\psi$ is not a substitution and therefore not necessarily a homomorphism. The next series of lemmas investigate those situations in which $\psi(w)=\psi(w)$, since this equality has important consequences.

(5.25) REMARK. Let $\langle\Omega, X\rangle$ be a free universal algebra. We suppose that a notion of depth of a word $w \in\langle\Omega, X\rangle$ has been defined such that the following are satisfied.

(i) For all $w$, depth $w$ is an ordinal number.

(ii) The range of depth $w$ is bounded by some ordinal $m=m(\Omega)$.

(iii) depth $w=0$ if and only if $w \in X$ or $w=\omega$ for some nullary operation $w \in \Omega$.

(iv) If $w=\omega(v)$ for some $\mathbf{v} \in\langle\Omega, X\rangle^{n}$ then depth $w=$ depth $v_{i}$ for all $i<n$.

If $\Omega$ is finitary the number of symbols in $w$ minus one will serve as depth $w$. In any case such a function is constructed in $\S 7$.

(5.26) LEMMA. Let $\theta, \psi$ be substitutions from $\langle\Omega, X\rangle$ into the partially ordered universal algebra $(\Omega, T)$. If for all $x \in X, \theta(x) \leqq \psi(x)$, then for all $w \in\langle\Omega, X\rangle, \theta(w) \leqq$ $\leqq \psi(w)$.

Proof. Let $w \in\langle\Omega, X\rangle$. If depth $w=0$, the statement is trivial. Suppose that depth $w>0$ and that the result is true for all words of depth less than depth $w$. Let $w=\omega(\mathbf{u})$. Then depth $u_{i}<$ depth $w, i<n$ and so by the inductive hypothesis $\theta\left(\mathbf{u}_{i}\right) \leqq$ $\leqq \psi\left(\mathbf{u}_{i}\right)$. Hence $\theta(w)=\omega(\theta(\mathbf{u})) \leqq \omega(\varphi(\mathbf{u}))=\varphi(w)$.

(5. 27) Proposition. Let $\left(^{*}, \Omega, T\right)$ be a superior completion of $(\Omega, S)$, a partially ordered universal algebra. Let $w \in\langle\Omega, X\rangle$. Then for every substitution $\psi$ of $X$ into $T, \psi(w) \leqq \psi(w)$.

Proof. Let $\theta$ and $\psi$ be substitutions from $X$ into $T$ and suppose that $\theta(x) \leqq$ $\leqq \psi(x)$, all $x \in X$. Then by $(5.26), \theta(w) \leqq \psi(w)$, all $w \in\langle\Omega, X\rangle$. Hence $\psi(w)=$ $=\sup \{\theta(w): \theta(x) \in L(S, \psi(x))\} \leqq \psi(w)$.

(5.28) Proposition. 'Let $(\Omega, S)$ be a partially ordered universal algebra, and let $(*, \Omega, T)$ be a suprema preserving superior completion of $(\Omega, S)$. If $w$ is a word in $\langle\Omega, X\rangle$ such that each $x \in X$ occurs in $w$ at most once, then, for each substitution $\psi$ of $X$ into $T, \psi(w)=\psi(w)$.

Proof. The proof is by induction on the depth of the word $w$. If depth $w=0$ there are two cases: Either $w=\omega$, with $\omega$ nullary, in which case $\psi(w)=\omega(\varnothing)=\psi(w)$, or $w=x \in X$, in which case $\psi(w)=\sup \{\theta(x): \theta(x) \in L(S, \psi(x))\}=\sup L(S, \psi(x))=$ $=\psi(x)=\psi(w)$. Suppose inductively that depth $w \geqq 1$, and suppose the proposition 
true for words of depth less than depth $w$. Let $w=\omega(\mathbf{u})$. Then since $\psi$ is a homomorphism and by induction

$$
\psi(w)=\omega(\psi(\mathbf{u}))=\omega(\psi(\mathbf{u})) .
$$

Let $A_{i}=\left\{\theta\left(w_{i}\right): \theta(x) \in L(S, \psi(x))\right\}$. Then by definition of $\psi$ and since $(*, \Omega, T)$ preserves suprema

$$
\omega(\psi(\mathbf{u}))=\omega(\sup \mathbf{A})=\sup \bar{\omega}(\mathbf{A})=\sup \left\{\omega(\mathbf{a}): a_{i} \in A_{i}\right\} .
$$

Let $a_{i} \in A_{i}$. Then there exists $\theta_{i}$ such that $\theta_{i}(x) \in L(S, \psi(x))$ and $\theta_{i}\left(u_{i}\right)=a_{i}$. Since no $x$ occurs in more than one $u_{i}, i<n$ we may define a substitution $\theta$ of $X$ into $S$ by

$$
\theta(x)= \begin{cases}\theta_{i}(x), & \text { if } x \text { occurs in } u_{i}, \quad i<n \\ \theta_{1}(x) & \text { otherwise. }\end{cases}
$$

Note that $\theta(x) \in L(S, \psi(x))$ since $\theta_{i}(x) \in L(S, \psi(x))$. Thus $\theta\left(u_{i}\right)=a_{i}$, and we obtain

$$
\begin{gathered}
\sup \left\{\omega(\mathbf{a}): a_{i} \in A_{i}\right\}=\sup \{\omega(\theta(\mathbf{u}): \theta(x) \in L(S, \psi(x))\}= \\
=\sup \{\theta(\mathbf{w}): \theta(x) \in L(S, \psi(x))\}=\psi(w) .
\end{gathered}
$$

The equality now follows from (a), (b), and (c).

(5.29) Definttion. Let $w_{1}, w_{2} \in\langle\Omega, X\rangle$. We say (the equality) $w_{1} \equiv w_{2}$ ((the inequality) $\left.w_{1} \ll w_{2}\right)$ holds identically on the universal algebra $(\Omega, A)$ if and only if, for all substitutions $\psi$ of $X$ into $A, \psi\left(w_{1}\right)=\psi\left(w_{2}\right)\left(\psi\left(w_{1}\right) \leqq \psi\left(w_{2}\right)\right)$.

(5.30) THEOREM. Let $(\Omega, S)$ be a partially ordered universal algebra, and let $(*, \Omega, T)$ be a superior completion of $(\Omega, S)$ which is suprema preserving. Let $w_{1}, w_{2} \epsilon$ $\epsilon\langle\Omega, X\rangle$ and suppose each $x \in X$ occurs at most once in each $w_{i}, i=1,2$. Then $w_{1} \equiv w_{2}$ $\left(w_{1} \ll w_{2}\right)$ holds identically on $(\Omega, S)$, if and only if it holds on $(*, \Omega, T)$.

PROoF. It is sufficient to prove the theorem $w_{1} \ll w_{2}$ since $w_{1} \equiv w_{2}$ is equivalent to $w_{1} \ll w_{2}$ and $w_{2} \ll w_{1}$. If $w_{1} \equiv w_{2}$ holds on $(*, \Omega, T)$ then it clearly holds on the subalgebra $(\Omega, S)$. Conversely, suppose $w_{1} \ll w_{2}$ holds on $(\Omega, S)$. Let $\psi$ be a substitution of $X$ into $T$. Then by (5.28) and the definition of $\psi$

$$
\psi\left(w_{i}\right)=\psi\left(w_{i}\right)=\sup \left\{\theta\left(w_{i}\right): \theta(x) \in L(\psi(x))\right\} .
$$

But, since $w_{1} \ll w_{2}$ on $S, \theta\left(w_{1}\right) \leqq \theta\left(w_{2}\right)$ for all substitutions $\theta$, and the result follows.

(5.31) Corollary. Let $w_{1}, w_{2} \in\langle\Omega, X\rangle$ and suppose each $x \in X$ occurs at most once in each $w_{i}, i=1,2$. Then $w_{1} \equiv w_{2}\left(w_{1} \ll w_{2}\right)$ holds identically on $(\Omega, S)$ if and only. if it holds on $(*, \Omega, \mathscr{I}(S))$.

Proof. Immediate by Theorem (5.13).

(5.32) CoRollary. Let $(., S)$ be a groupoid. If $(., S)$ has any of the following properties, then so has every superior completion of $(., S)$ which preserves suprema, in particular $(*, ., \mathscr{I}(S))$ : (1) associativity, (2) commutativity, (3) existence of identity, (4) existence of zero.

Proof. This is an immediate consequence of Theorem (5.30). We note that in the case of (3) and (4) we have to adjoin constant nullary operations to $\Omega$. 
(5. 33) EXAMPLE. In general, idempotence, distributivity, power associativity and inversion in $S$ do not necessarily imply their counterparts in $\mathscr{I}(S)$. For example, let $S=\{a, b, c\}$ with the trivial ordering in which distinct elements are incomparable. Define $u v=u, u+u=u$ and for $u \neq v, u+v=w \&\{u, v\}$. Then in $S, x+x \equiv x$ and $x(y+z) \equiv x y+x z$ hold identically, but neither holds in $\mathscr{I}(S)$. We have been unable to characterize the class of identities which for every $\Omega$ algebra $(\Omega, S)$ extend $(*, \Omega, \mathscr{I}(S))$ whenever they hold in $(\Omega, S)$.

We recall $\Im(S)$ was defined in $(3.22)$.

(5.34) THEOREM. Let $(\Omega . S)$ be a partially ordered universal algebra. Then the restriction of $\left({ }^{*}, \Omega, \mathscr{I}(S)\right)$ to $\mathfrak{I}(S)$ is a subalgebra and is, in fact, the superior. completion $(*, \Omega, \mathfrak{I}(S))$ as constructed (5.6). Further, $(*, \Omega, \Im(S))$ is suprema preserving.

Proof. Let $w \in \Omega$ be $n$-ary. Let $\mathbf{A} \in \mathfrak{I}(S)^{n}$. We wish to show that ${ }^{*} \omega(\mathbf{A}) \in \mathfrak{I}(S)$, where ${ }^{*} \omega$ is the operation in $(*, \Omega, \mathscr{I}(S))$. Since $A_{i} \in \mathfrak{J}(S), i<n$ there exists $s_{i} \in S_{i}$ such that $A_{i} \subseteq l\left(s_{i}\right), i=1, \ldots, n$. Hence

whence ${ }^{*} \omega(\mathbf{A}) \in \mathfrak{J}(S)$.

$$
{ }^{*} \omega(\mathbf{A}) \leqq * \omega(\iota(\mathbf{s}))=\iota(\omega(\mathbf{s}) \in \iota(S),
$$

Hence the restriction of $(*, \Omega, \mathscr{I}(S))$ to $\Im(S)$ is a subalgebra. Further if $\mathscr{A} \subseteq \Im(S)$, and sup $\mathscr{A}$ exists in $\mathfrak{I}(S)$, then the supremum in $\mathfrak{I}(S)$ is the same as the supremum in $\mathscr{I}(S)$, by (3.23). Hence the subalgebra is suprema preserving since, by (5.13), $(, * \Omega, \mathscr{I}(S))$ is. It follows from (5.15) that the subalgebra is the algebra constructed on $\mathfrak{J}(S)$ by $(5,6)$.

(5. 35) COROLlary. Let $(\Omega, S)$ be a partially ordered universal algebra. Then $(*, \Omega, \Im(S))$ is a suprema preserving superior completion of $(\Omega, S)$ which further satisfies: If $w_{1}, w_{2} \in\langle\Omega, X\rangle$ are words such that each $x \in X$ occurs at most once in each $w_{i}, i=1,2$, then $w_{1} \equiv w_{2}\left(w_{1} \ll w_{2}\right)$ holds identically in $(\Omega, S)$ if and only if it holds in $(*, \Omega, \Im(S))$.

Proof. The first part of the corollary follows from (5.34), while the second part follows from (5.31), and the facts that $(*, \Omega, \Im(S))$ is an extension of $(\Omega, S)$ and a subalgebra of $(*, \Omega, \mathscr{I}(S))$.

\section{Linearly ordered universal algebras}

We now consider the case when $(\Omega, S)$ is a linearly ordered universal algebra. In this case theorem (5.30) can be strengthened. We begin with a strengthened version of (5.28).

(6. 1) Proposition. Let $(*, \Omega, T)$ be a suprema preserving superior completion of the linearly ordered universal algebra $(\Omega, S)$. If $w \in\langle\Omega, X\rangle$ then for each substitution $\psi$ of $X$ into $T, \psi(w)=\psi(w)$.

Proof. The proof is the same as that of proposition (5.28), except that the substitution $\theta$ is now defined as follows:

$$
\theta(x)=\max \left\{\theta_{i}(x): i<n\right\} .
$$


We note that $\theta(x)$ is well-defined since $S$ is linearly ordered. Since for each $x$ there is an $i, i<n$, such that $\theta(x)=\theta_{i}(x)$, it follows that $\theta(x) \in L(S, \psi(x))$.

(6.2) THEOREM. Let $\left({ }^{*}, \Omega, T\right.$ ) be a suprema preserving superior completion of the linearly ordered universal algebra $(\Omega, S)$. Let $w_{1}, w_{2} \in\langle\Omega, X\rangle$. Then $w_{1} \equiv w_{2}$ $\left(w_{1} \ll w_{2}\right)$ holds on $(\Omega, S)$ if and only if it holds identically on $(*, \Omega, T)$.

PROOF. The proof is similar to the proof of theorem (5.30), except that we use (6.1) in place of (5.28).

(6.3) Corollary. Let $(., S)$ be a linearly ordered groupoid. If $(., S)$ has any of the properties of (5.32), or any of the properties below, then so has every superior completion of $(., S)$ which preserves suprema, in particular $(*, ., \mathscr{I}(S))$ : (5) idempotence, (6) power associativity.

(6. 4) REMARKs. (1) Let $(., G)=G$ be any partially ordered group with more than one element. Then $\mathscr{I}(G)$ is not group, since $G \in \mathscr{I}(G)$, and $G$ has no inverse in $\mathscr{I}(G)$.

(2) The class of all groups may be defined to be the primitive class (KUROSH [5], $\S 22.1)$ or universal algebras with a binary opesation $\omega_{2}(a, b)=a b$, a unary operation $\omega_{1}(a)=a^{-1}$, and a nullary operation $\omega_{0}=1$, which satisfy the identities $x(y z) \equiv(x y) z, x 1 \equiv x$ and $x x^{-1} \equiv 1$. There are other equivalent ways of defining the class of groups by means of operations and identities (KUROSH [5], § 18.6). Let $\Lambda$ be a set of identities with words taken from $\langle\Omega, X\rangle$, so that the class. of universal algebras $\left({ }^{\prime}, \Omega, G\right)$ satisfying the identities $\Lambda$ is the class of groups.

There exists an identity $w_{1} \equiv w_{2}$ in $A$ such that some $x \in X$ occurs at least twice in either $w_{1}$ or $w_{2}$. For, optherwise, let $\left({ }^{\prime}, \Omega, G\right)$ be a group with trivial ordering, and more than one element. Then, by $(5.30)(*, \Omega, \mathscr{I}(G))$ would be a group contrary to (1) above. Further, it is impossible to find a linear ordering on any group $G$ so that every $\omega \in \Omega$ is order preserving on $G^{n}$. For, otherwise, $(*, \Omega, \mathscr{I}(G)$ ) would be a group by (6.2), again contrary to (1) above. In particular the unary operation $\omega_{1}(a)=a^{-1}$, is order inverting. Similar remarks may be made about the classes of quasi-groups and loops.

(6. 5) Definitions. Let $(\Omega, S)$ be a linearly ordered universal algebra, and let $\omega \in \Omega$. We say that $\omega$ is lower semi-continuous if and only if $\omega$ satisfies the following condition:

If $\omega(\mathbf{a})>c$ then there exist neighborhoods $N\left(a_{i}\right)$ of $a_{i}$ (in the order topology) such that for $b_{i} \in N\left(a_{i}\right), \omega(\mathbf{b})>c$.

We call the algebra $(\Omega, S)$ lower semi-continuous if each $w \in \Omega$ is lower semicontinuous. Upper semi-continuity for operations (algebras) is defined dually. If an operation (algebra) is both lower and upper semi-continuous, then it is called continuous. The following theorem is essentially due to A. H. CLIFFord ([1], Lemma 2. 1) who proved it in the case of commutative semigroups (cf. FucHs [2] p. 176, who proved it for arbitrary semigroups).

(6.6) Theorem. Let $(\Omega, S)$ be a linearly ordered universal algebra, and let $\omega \in \Omega$. Then $\omega$ is lower semi-continuous if and only if $\omega$ is suprema preserving.

Proof. The proof can be obtained by making the appropriate, obvious modifications of CLIFFIORD's proof. 
(6.7) Corollary. Let $(\Omega, S)$ be a linearly ordered universal algebra. Then $(\Omega, S)$ is lower semi-continuous if and only if it is supprema preserving.

(6.8) THEOREM. Let $(\Omega, S)$ be a linearly ordered universal algebra. Then both $(*, \Omega, \mathscr{I}(S))$ and $(*, \Omega, \Im(S))$ are lower semi-continuous completions of $S$.

ProOr. By (3.13) $\mathscr{I}(S)$ is a superior completion of $S$, which is linearly ordered by (2. 11). The conclusion follows from (5. 13), (5. 34) and ClifFord's theorem (6. 6).

(6.9) Theorem. Let $(\Omega, S)$ be a linearly ordered universal algebra. Then there exist linearly ordered completions $\left({ }^{\prime}, \Omega, \mathscr{F} \mathscr{I}(S)\right)$ and $\left({ }^{\prime \prime}, \Omega, \mathscr{F} \mathscr{I}(S)\right)$ which are upper and lower semi-continuous respectively. Further an inequality (equality) holds identically on $\left({ }^{\prime}, \Omega, \mathscr{F} \mathscr{I}(S)\right)((", \Omega, \mathscr{F} \mathscr{I}(S)))$ if and only if it holds on $(\Omega, S)$.

Proof. By (5.13) and (6. 2), $(\Omega, S)$ can be extended to $\mathscr{I}(S)$ so that ,identities" are preserved. By $(5.13)^{*}$ and $(6.2)^{*}$, this algebra on $\mathscr{I}(S)$ can be extended to algebras $\left(', \Omega, \mathscr{F} \mathscr{I}(S)\right.$ ), which are upper semi-continuous by $(6.7)^{*}$, and in which all identities on $\mathscr{I}(S)$ and hence on $S$, remain valid.

Dually, we prove the corresponding result for a lower semi-continuous extension $(", \Omega, \mathscr{I} \mathscr{F}(S))$. But $\mathscr{I} \mathscr{F}(S)$ is order isomorphic to $\mathscr{F} \mathscr{I}(S)$ by $(4.22)$ and hence we may replace $\mathscr{I} \mathscr{F}(S)$ by $\mathscr{F} \mathscr{I}(S)$ in this algebra.

(6.10) REMARK. In theorem (6.9), we may replace $\mathscr{F} \mathscr{I}(S)$ by $\mathscr{F} \mathfrak{I}(S)$. This follows from (5.34).

(6. 11) REMARK. It is usually impossible to extend the operations of $(\Omega, S)$ to $\mathscr{F} \mathscr{I}(S)$ (or $\mathscr{F} \Im(S)$ ) so that the resulting algebra is continuous. The example is even more surprising, since in the counter-example below $(\Omega, S)=(+, R)$ is the reals under addition. Then $\mathfrak{\Im} \Im(R)=R \cup \pi^{+}(R) \cup \pi^{-}(R)$. Let $0^{+}$and $0^{-}$be the predecessor and successor respectively of 0 in $\widetilde{\Im}(R)$. We suppose that addition is continuous in $\mathfrak{F} \Im(R)$, and we derive a contradiction.

If $x \in R$, and $x>0$, then $x+0^{-}>x+\left(-\frac{x}{2}\right)=x / 2$. Since $0^{+}=\inf U^{\#}(R, 0)$ we obtain, by continuity, $0^{+}+0^{-}=\inf \left\{x+0^{-}: x \in U_{\#}(R, 0)\right\}=0^{+}$. Dually, $0^{+}+0^{-}=0^{-}$, and this is a contradiction.

(6. 12) ThEorem. Let $(\Omega, S)$ be a linearly ordered universal algebra, and let $N$ be a normal extension of $S$ such that $(*, \Omega, N)$ exists. Then $(*, \Omega, N)$ is lower semicontinuous if and only if $(\Omega, S)$ is lower semi-continuous.

Proof. If $(*, \Omega, N)$ is lower semi-continuous, so is $(\Omega, S)$ by $(5.17)$ and $(6.6)$. Let $s \in S, x \in N, x<s$. We claim there exists $c \in S$ such that $x \leqq c<s$, since $x=\inf L(S, x)$. Let $\mathbf{t} \in N^{n}$, suppose $\omega \in \Omega$, and $x<^{*} \omega(\mathbf{t})$. By definition of ${ }^{*} \omega$, there exist $\mathbf{s} \in S^{n}$ with $\mathbf{s} \leqq \mathbf{t}$ for which $x<\omega(\mathbf{s})$. Hence, for some $c \in S, x \leqq c<\omega(\mathbf{s})$. Since $\omega$ is lower semi-continuous, there exist $\mathbf{s}^{\prime} \in S$ such that for all $\mathbf{u}>\mathbf{s}^{\prime} \omega(u)>c$. Let $\mathbf{v} \in N^{n}, v_{i}>s_{i}^{\prime}$, $i<n$. Since $N$ is a superior extension of $S$, there exist $\mathbf{u} \in S^{n}$ for which $v_{i} \geqq u_{i}>s_{i}^{\prime}$, whenc $* \omega(\mathbf{v}) \geqq \omega(\mathbf{u})>c \geqq x$ and hence ${ }^{*} \omega$ is lower semi-continuous.

(6. 13) THEOREM. Let $(\Omega, S)$ be a linearly ordered continuous universal algebra and let $N$ be the normal completion of $S$. Then

(1) $(*, \Omega, N)$ exists.

(2) (*, $\Omega, N)$ is the minimal extension of $(\Omega, S)$ in the ordering (5. 14).

(3) $(*, \Omega, N)$ is lower semi-continuous. 
Proof. The extension (*, $\Omega, N)$ exists by (4.27) and (5.34). The theorem follows from (6. 12).

(6. 14) THEOREM. Let $(\Omega, S)$ be a continuous linearly ordered universal algebra. Let $A$ be a set of equalities and inequalities which hold identically on $(\Omega, S)$. Let $N$ be the normal completion of $S$. Then the following are equivalent: cally.

(i) There exists a continuous completion of $(\Omega, S)$ to $N$ in which $A$ holds identi-

(ii) There exists a continuous completion of $(\Omega, S)$ to $N$,

(iii) There exists a unique completion of $(\Omega, S)$ to $N$.

(iv) There exists a unique completion of $(\Omega, S)$ to $N$ in which $\Lambda$ holds identically.

Proof. Clearly (i) implies (ii). From (5.19), (6.8) and (6.8)* we infer that (ii) implies (iii). Suppose (iii) holds. By (6. 13), the unique completion is $(*, \Omega, N)$, and (iv) follows from (6.2). Suppose (iv) holds. From (6.13.1) and (6.13.1)* we see that $(*, \Omega, N)=(*, \Omega, N)$ is the unique completion. Again by $(6.13 .3)$ and $(6.13: 3)^{*}$, (i) follows.

(6.15) REMARK. In the case when $\Omega$ consists of a single binary operation $\omega(a, b)=a b$ and $A$ consists of the identities $x y=y x$ and $x(y z)=(x y) z$, i.e. $S$ is a commutative semigroup, the equivalence of (i) and (iv) of (6.11) was first shown by ClIFFORD [1].

\section{Free infinitary algebras}

In this section we construct the free universal algebra with operations $\Omega$ generated by $X$ and show that a depth function (cf. 5. 25) can be defined.

(7. 1) Remarks. (1) We shall follow the definitions of cardinal numbers and ordinal numbers given by GöDeL [4]. In particular an ordinal number is a set which is an initial segments of the class of ordinals which is naturally well ordered by $\epsilon$, and an ordinal number is, in fact, the set of ordinals less than it. A cardinal number is an ordinal number which is less than all other ordinal numbers cardinality equivalent to it, and hence a limit ordinal, if infinite.

(2) We shall also use the result that if $\delta$ is an infinite cardinal number and $A$ is a set of ordinal numbers each less than $\delta$, and the cardinality of $A$ is also less than $\delta$, then $\sup A=\cup A<\delta$.

(7. 2) Definitrons. (1) By a set of operations $\Omega$ we mean an ordered pair $(\Omega, n)$, where $n$ is a function from $\Omega$ into an infinite cardinal number $m=m(\Omega)$. We normally write $\Omega$ for $(\Omega, n)$.

(2) Let $\Omega$ be a set of operations, and $X$ a set disjoint from $\Omega$. Let $\Omega_{0}=$ $=\left\{\omega \in \Omega_{0}: n(\omega)=0\right\}$.

(i) Let $\mathscr{W}_{0}=X \cup \Omega_{0}$.

(ii) If $\mathscr{W}_{i}$ has been defined for all ordinals $i$ less than $j$, we define $\mathscr{W}_{j}$ thus: Let $\mathscr{V}_{j}=\bigcup\left\{\mathscr{W}_{i}: i<j\right\}, \mathscr{W}_{j}=\left\{(\omega, \nabla): \omega \in \Omega, \nabla \in \mathscr{V}_{j}, n=n(\omega)\right\} \cup \mathscr{V}$.

(7.3) Lemma. If $m$ is the cardinal number defined in (7.2.1), then $\mathscr{W}_{m}=\mathscr{V}_{m}$.

Proof. Clearly $\mathscr{W}_{m} \supseteq \mathscr{V}_{m}$. Let $w \in \mathscr{W}_{m}$. If $w \in \mathscr{W}_{0}$, then $w \in \mathscr{V}_{m}$. Otherwise $w=(\omega, v)$ where $v \in \mathscr{V}_{m}^{n}$. Thus, $v_{i} \in \mathscr{W}_{j(i)}$ for some ordinal $j(i)<m$, for all $i<n=n(\omega)$. 
Let $j=\sup \{j(i): i<n\}$. Since each $j(i)$ is less than $m$ and $n<m$ we see by (7.1.2) that $j<m$. Thus for all $i<n, v_{i} \in \mathscr{W}_{j} \subseteq \mathscr{V}_{j+1}$. It follows that $w \in \mathscr{W}_{j+1}$, and since $j<m$ and $m$ is a limit ordinal, $j+1<m$. Thus $\mathscr{W}_{j+1} \subseteq \mathscr{V}_{m}$ whence $w \in \mathscr{V}_{m}$. The result follows.

(7. 4) Corollary. $\mathscr{W}_{m+1}=\mathscr{W}_{m}$.

(7. 5) Drfinitions. (1) Let $\langle\Omega, X\rangle=\mathscr{W}_{m}$. This set is called the set (of words) of the free universal algebra generated by $\Omega$ and $X$.

(2) The free universal algebra generated by $\Omega$ and $X$ is the triple $\left({ }^{\prime}, \Omega,\langle\Omega, X\rangle\right)$ where ' $\omega$ is the function from $\langle\Omega, X\rangle^{n}$ to $\langle\Omega, X\rangle$ given by ${ }^{\prime} \omega(\mathbf{v})=(\omega, \mathbf{v})$, where $n=n(\omega)$.

In practice we call $\langle\Omega, X\rangle$ the free algebra.

(7.6) Definition. If $w \in\langle\Omega, X\rangle$ then we define $\operatorname{depth} w=\inf \left\{i: w \in \mathscr{W}_{i}\right\}$.

We observe that depth $w$ has all the properties required in (5. 25).

(Received 17 August 1965)

\section{References}

[1] A. H. Clifford, Completion of totally ordered commutative semigroups, Duke Math. J., 26 (1959), pp. 41-59.

[2] P. M. CoHn, Universal Algebra (Harper and Row, New York, 1965).

[3] L. FucHs, Partially ordered algebraic systems (Addison-Wesley, Reading, 1963).

[4] K. GöDEL, The consistency of the continuum hypothesis, Annals. Math. Studies, Princeton $U .,(1940)$.

[5] J. L. Kelley, General Topology (Van Nostrand, Princeton, 1955).

[6] A. G. Kurost, General Algebra (Chelsea, New York, 1963). 